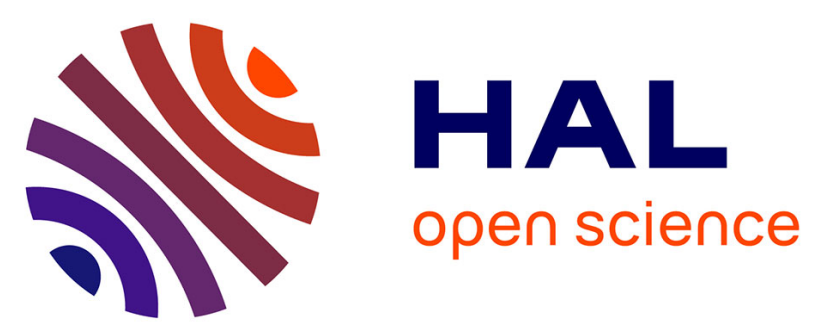

\title{
Discontinuous Galerkin method in time combined with a stabilized finite element method in space for linear first-order PDEs
}

\author{
Alexandre Ern, Friedhelm Schieweck
}

\section{- To cite this version:}

Alexandre Ern, Friedhelm Schieweck. Discontinuous Galerkin method in time combined with a stabilized finite element method in space for linear first-order PDEs. Mathematics of Computation, 2016, 85 (301), pp.2099-2129. hal-00947695v1

\section{HAL Id: hal-00947695 \\ https://hal.science/hal-00947695v1}

Submitted on 17 Feb 2014 (v1), last revised 20 Jan 2018 (v2)

HAL is a multi-disciplinary open access archive for the deposit and dissemination of scientific research documents, whether they are published or not. The documents may come from teaching and research institutions in France or abroad, or from public or private research centers.
L'archive ouverte pluridisciplinaire HAL, est destinée au dépôt et à la diffusion de documents scientifiques de niveau recherche, publiés ou non, émanant des établissements d'enseignement et de recherche français ou étrangers, des laboratoires publics ou privés. 


\title{
Discontinuous Galerkin method in time combined with a stabilized finite element method in space for linear first-order PDEs
}

\author{
Alexandre Ern*, Friedhelm Schieweck ${ }^{\dagger}$
}

February 17, 2014

\begin{abstract}
We analyze the discontinuous Galerkin method in time combined with a finite element method with symmetric stabilization in space to approximate evolution problems with a linear, first-order differential operator. A unified analysis is presented for space discretization, including the discontinuous Galerkin method and $H^{1}$-conforming finite elements with interior penalty on gradient jumps. Our main results are error estimates in various norms for smooth solutions. Two key ingredients are the post-processing of the fully discrete solution by lifting its jumps in time and a new time-interpolate of the exact solution. We first analyze the $L^{\infty}\left(L^{2}\right)$ and $L^{2}\left(L^{2}\right)$ errors and derive a super-convergent bound of order $\left(\tau^{k+2}+h^{r+1 / 2}\right)$ in the case of static meshes for $k \geq 1$. Here, $\tau$ is the time step, $k$ the polynomial order in time, $h$ the size of the space mesh, and $r$ the polynomial order in space. For the case of dynamically changing meshes, we derive a novel bound on the resulting projection error. Finally, we prove new optimal bounds on static meshes for the error in the time-derivative and in the discrete graph norm.
\end{abstract}

Keywords: discontinuous Galerkin in time, stabilized FEM, first-order PDEs, graph norm error estimates, superconvergence, dynamic meshes

2000 Mathematics Subject Classification (MSC): 65M12, 65M60, 65J10

\section{Introduction}

Our goal is to analyze the discontinuous Galerkin $(\mathrm{dG})$ method in time combined with a finite element method (FEM) with symmetric stabilization in space to approximate the linear evolution problem

$$
\partial_{t} u+A u=f \quad \text { in } \Omega \times(0, T),
$$

completed with suitable boundary and initial conditions, see Section 2 . Here, $\Omega$ is a domain in $\mathbb{R}^{d}, d \geq 1, T$ some positive final time, $f$ a source term, and $A$ some linear, time-independent,

\footnotetext{
${ }^{*}$ Universite Paris-Est, CERMICS (ENPC), 77455 Marne la Vallee Cedex 2, France, Email: ern@cermics.enpc.fr

${ }^{\dagger}$ Institut für Analysis und Numerik, Otto-von-Guericke-Universität Magdeburg, Postfach 4120, D-39016 Magdeburg, Germany, Email: schiewec@ovgu.de
} 
first-order differential operator in space. We assume for simplicity that $A$ is an advectionreaction operator; more generally, $A$ can be a Friedrichs' operator, whereby (1) is a system of first-order PDEs endowed with a symmetry and a positivity property [21, 19].

The dG method in time can be used, in the spirit of Rothe's method, to semi-discretize in time the evolution problem (1). This method uses piecewise polynomial ansatz and trial spaces of some order $k \geq 0$, whose elements are $V$-valued functions of time that can be discontinuous at the discrete nodes defining the time partition; here, $V$ denotes the graph space associated with the space differential operator $A$. The time semi-discrete problem can then be discretized in space by a stabilized FEM. A relatively wide class of stabilized FEM is that based on symmetric stabilization, including on the one hand the dG method in space $[30,29,16]$ and on the other hand $H^{1}$-conforming finite elements with various stabilization techniques, e.g., interior penalty on gradient jumps $[6,9]$, local projection $[4,34]$, subgrid viscosity $[22,23]$, or orthogonal subscales $[14,15]$. When used to approximate the steady version of (1), all of these methods lead to quasi-optimal $L^{2}$-error estimates of order $h^{r+1 / 2}$, where $h$ denotes the size of the space mesh and $r$ the polynomial order in space, and optimal error estimates in the discrete graph norm. In what follows, we consider a unified analysis for a discrete differential operator $A_{h}$ satisfying three design properties (consistency, stability, and boundedness). These properties cover, as main examples, dG methods and $H^{1}$-conforming finite elements with interior penalty on gradient jumps. Minor modifications are to be included for other (weakly) non-consistent stabilization techniques.

The dG method in time has been extensively studied in [37, 17], see also references therein, for linear parabolic problems, either as time semi-discretization method in the Hilbert space $H^{1}$ or combined with $H^{1}$-conforming finite elements for space discretization. One of the main results for linear parabolic problems with a symmetric coercive operator $A$ is superconvergence in $L^{\infty}\left(L^{2}\right)\left(L^{\infty}\right.$ in time and $L^{2}$ in space) and $L^{2}\left(L^{2}\right)$ norms of order $\left(\tau^{2 k+1}+h^{r+1}\right)$ where $\tau$ is the time step and $h$ the space mesh size. Further results on the dG method in time, combined with $\mathrm{dG}$ methods in space, concern the convergence to entropy solutions for hyperbolic conservation laws [28], the $h p$-analysis for diffusion and incompressible flow problems [35, 39], and inviscid compressible flows [38]. $L^{\infty}\left(L^{2}\right)$ and $L^{2}\left(L^{2}\right)$ error estimates of order $\left(\tau^{k+1}+h^{r}\right)$ are derived in $[20,10]$ for nonlinear convection-diffusion problems on time-varying meshes under the assumption $h^{2} \lesssim \tau$. Finally, we mention the work [2] for linear transient convection-diffusion-reaction problems on static meshes where the dG method in time is combined with local projection stabilization in space for $H^{1}$-conforming finite elements leading to an $L^{\infty}\left(L^{2}\right)$ and $L^{2}\left(L^{2}\right)$ error bound of order $\left(\tau^{k+1}+h^{r+1 / 2}\right)$ if the diffusion parameter $\varepsilon$ is less than $h$.

An alternative approach for discretizating the evolution problem (1) is the explicit RungeKutta (RK) method in time combined with the dG method in space and suitable limiters, see $[13,12,11]$. Since the time-stepping scheme is explicit, such methods are computationally effective, but are only conditionally stable, and the error bounds require the application of the Gronwall argument which implies that the error constant grows exponentially with respect to the final time $T$. The analysis of explicit RK methods combined with stabilized FEM entails some subtleties. Error estimates are available for second-order (RK2) and third- 
order (RK3) methods; see [42, 43] for nonlinear conservation laws and dG methods in space and [8] for Friedrichs' systems and FEM with symmetric stabilization. The main results are $L^{\infty}\left(L^{2}\right)$ error estimates of order $\left(\tau^{2}+h^{r+1 / 2}\right)$ for RK2 under a tightened 4/3-CFL condition (except for piecewise affine polynomials in space where the usual CFL condition suffices) and of order $\left(\tau^{3}+h^{r+1 / 2}\right)$ for RK3 under the usual CFL condition. In contrast to explicit RK methods, the dG method in time is unconditionally stable, and leads, in some cases, to super-convergent error estimates. The prize to pay is obviously increased computational cost, although this drawback can be tamed using efficient multigrid solvers, as in $[25,27,26]$ for the heat, Stokes, and Navier-Stokes equations. Antoher advantage of dG methods in time is that their analysis readily encompasses all polynomial orders $k \geq 1$ in time (the lowest-order case $k=0$, corresponding to the implicit Euler scheme, being slightly different). In addition, the derived error bounds do not require the Gronwall argument, and the error constant grows only like $T^{1 / 2}$ with respect to the final time $T$. Moreover, since they hinge on the weak form of the evolution problem, dG methods in time can be cast more effectively into optimization problems constrained by time-dependent PDEs [36, 31, 33]. Finally, we mention the recent analysis of implicit RK methods in time combined with $\mathrm{dG}$ methods in space for linear Maxwell's equations, leading in particular to an $L^{\infty}\left(L^{2}\right)$ error estimate of order $\left(\tau^{s+1}+h^{r+1 / 2}\right)$ where $s$ is the number of distinct time nodes in the RK method [24].

The main contributions of this paper are as follows. Let $u$ be the exact solution and let $u_{\tau h}$ be the fully discrete solution. We analyze the error between the exact solution and a post-processed discrete solution $\tilde{u}_{\tau h}=\mathcal{L}_{\tau} u_{\tau h}$ which is continuous in time and is a piecewise polynomial in time of order $(k+1)$. The operator $\mathcal{L}_{\tau}$, motivated by [32] where the link between the $\mathrm{dG}$ method in time of order $k$ and a continuous Petrov-Galerkin method of order $(k+1)$ with reduced integration is explored, is the first key ingredient of our analysis. The second one is a new time-interpolate $R_{\tau}^{k+1} u$ of the exact solution $u$ of order $(k+1)$, which, in particular, interpolates at the $(k+1)$ right-sided Gauss-Radau integration points the time-derivative of a $C^{1}$-interpolate of order $(k+2)$ of the exact solution. Our first main result, which improves on the current state-of-the-art, are estimates for the error $\left(u-\tilde{u}_{\tau h}\right)$ in $L^{\infty}\left(L^{2}\right)$ at the discrete nodes defining the time partition and in $L^{2}\left(L^{2}\right)$, both of order $\left(\tau^{k+2}+h^{r+1 / 2}\right)$. This result implies, in particular, a super-convergent estimate of the same order for the error $\left(u-u_{\tau h}\right)$ at the discrete time nodes. The error estimates are presented first for static space meshes. For time-varying meshes, there is an additional projection error due to mesh changes, for which we derive a novel sharp estimate compared to existing results [40, 1, 20, 10]. Our second main result are estimates on the error derivatives; we focus on static space meshes for simplicity. To our knowledge, such estimates are not yet available even for static meshes. We emphasize that their derivation is more delicate than for parabolic PDEs since the differential operator $A$ is neither symmetric nor coercive in the graph norm. Our idea is to measure the error in the time-derivative using the post-processed time-derivative of the post-processed discrete solution, yielding a bound on $\left(\partial_{t} u-\mathcal{L}_{\tau} \partial_{t} \mathcal{L}_{\tau} u_{\tau h}\right)$ of order $\left(\tau^{k+1}+h^{r+1 / 2}\right)$ in $L^{\infty}\left(L^{2}\right)$ at the discrete time nodes and in $L^{2}\left(L^{2}\right)$. Finally, an optimal bound for the error $\left(u-\tilde{u}_{\tau h}\right)$ in the discrete graph norm is derived using the inf-sup stability of the discrete operator $A_{h}$.

The paper is organized as follows. Section 2 presents the continuous setting and Section 3 
the discrete setting in time and in space, including the lifting operator $\mathcal{L}_{\tau}$. Section 4 contains some preliminary results for the error analysis and, in particular, introduces the new timeinterpolate $R_{\tau}^{k+1} u$. Section 5 is devoted to the $L^{\infty}\left(L^{2}\right)$ and $L^{2}\left(L^{2}\right)$ error estimates. Finally, Section 6 deals with the estimates on the error derivatives.

\section{The continuous setting}

Let $\Omega \subset \mathbb{R}^{d}, d \geq 1$, be a domain with Lipschitz boundary $\Gamma$, and let $T$ be some positive final time. We consider the model problem: Find $u: \Omega \times[0, T] \rightarrow \mathbb{R}$ such that

$$
\begin{aligned}
\partial_{t} u+A u & =f & & \text { in } \Omega \times(0, T), \\
u & =0 & & \text { on } \Gamma_{-} \times(0, T), \\
u & =u_{0} & & \text { on } \Omega \times\{0\},
\end{aligned}
$$

where the linear first-order differential operator $A$ is of the form $A u:=\beta \cdot \nabla u+\sigma u, \beta: \Omega \rightarrow \mathbb{R}^{d}$ is a given Lipschitz convection field, $\sigma: \Omega \rightarrow \mathbb{R}$ a bounded reaction function, $f: \Omega \times[0, T] \rightarrow \mathbb{R}$ a source term, $u_{0}: \Omega \rightarrow \mathbb{R}$ a given initial value of $u$, and $\Gamma_{-}$(resp., $\Gamma_{+}$) the inflow (resp., outflow) part of the boundary defined as

$$
\Gamma_{ \pm}:=\{x \in \Gamma: \pm \beta(x) \cdot n>0\},
$$

with $n$ denoting the outer normal unit vector on $\Gamma$. We assume that the data $\beta$ and $\sigma$ are time-independent, and that

$$
\sigma(x)-\frac{1}{2} \operatorname{div} \beta(x) \geq \mu_{0}>0 \quad \forall x \in \Omega .
$$

The inner product in $L:=L^{2}(\Omega)$ is denoted $(\cdot, \cdot)_{L}$, and the norm $\|v\|_{L}^{2}:=(v, v)_{L}$. The graph space defined as $\{v \in L: \beta \cdot \nabla v \in L\}$ is a Hilbert space when equipped with the graph norm $\|v\|_{V}^{2}:=\mu_{0}\|v\|_{L}^{2}+\|\beta \cdot \nabla v\|_{L}^{2}$ (and the corresponding inner product). Assuming that $\Gamma_{-}$and $\Gamma_{+}$are well-separated, $V:=\left\{v \in L: \beta \cdot \nabla v \in L,\left.v\right|_{\Gamma_{-}}=0\right\}$ is a closed subspace of the graph space, and the operator $A: V \rightarrow L$ is an isomorphism; see, e.g., [19].

To characterize the smoothness of functions $t \mapsto v(t)$, we introduce, for a subinterval $J \subset[0, T]$, the space $C^{r}(J, B)$ spanned by $r$ times continuously differentiable, $B$-valued functions on $J$, where $B$ denotes a Banach space with norm $\|\cdot\|_{B}$ (typically, $B \in\{L, V\}$ ). The space $C^{r}(J, B)$ is equipped with the following norm and semi-norm:

$$
\|v\|_{C^{r}(J, B)}:=\max _{0 \leq k \leq r} \sup _{t \in J}\left\|\partial_{t}^{k} v(t)\right\|_{B}, \quad|v|_{C^{r}(J, B)}:=\sup _{t \in J}\left\|\partial_{t}^{r} v(t)\right\|_{B} .
$$

For a measurable subset $J \subset[0, T]$, we also use the Bochner space $L^{2}(J, B)$ defined as

$$
L^{2}(J, B):=\left\{v: J \rightarrow B:\|v\|_{L^{2}(J, B)}<\infty\right\} \quad \text { with } \quad\|v\|_{L^{2}(J, B)}:=\left(\int_{J}\|v(t)\|_{B}^{2} d t\right)^{1 / 2} .
$$

Now, assuming $f \in C^{0}([0, T], L)$ and $u_{0} \in V$, the model problem (2) can be written as the following linear evolution problem: Find $u \in C^{0}([0, T], V) \cap C^{1}([0, T], L)$ such that

$$
\begin{aligned}
\left(\partial_{t} u(t), v\right)_{L}+(A u(t), v)_{L} & =(f(t), v)_{L} \quad \forall v \in L, \quad \forall t \in(0, T), \\
u(0) & =u_{0} .
\end{aligned}
$$


The well-posedness of (4) results from the Hille-Yosida Theorem; see, e.g., [41, p. 248] or [18, p. 313].

\section{The discrete setting}

We proceed in the spirit of Rothe's method whereby the evolution problem (4) is first semidiscretized in time, leading to a sequence of discrete problems in a Hilbert space, which are then discretized in space. An important ingredient for the time semi-discretization is the lifting operator $\mathcal{L}_{\tau}$ introduced in Section 3.2. In what follows, for positive real numbers $a$ and $b, a \lesssim b$ stands for the inequality $a \leq C b$ with generic constant $C$ independent of the size of the space and time meshes, of the final time, and of the exact solution $u$; the value of $C$ can depend on the regularity of the spatial mesh, the polynomial degrees used for space and time discretization, and the model parameters (including $\Omega$ and the constant $\mu_{0}$ in (3)).

\subsection{Time semi-discretization by the $\mathrm{dG}(k)$-method}

In order to semi-discretize problem (4) in time, we decompose the time interval $I:=(0, T]$ into $N$ disjoint subintervals $I_{n}:=\left(t_{n-1}, t_{n}\right]$, where $n=1, \ldots, N$ and $0=t_{0}<t_{1}<\cdots<$ $t_{N-1}<t_{N}=T$, so that $I=\cup_{n=1}^{N} I_{n}$. Observe that all the time intervals are conventionally open at the left endpoint and closed at the right endpoint. In what follows, the maximum time step size $\tau:=\max _{1 \leq n \leq N} \tau_{n}$ where $\tau_{n}:=t_{n}-t_{n-1}$ is used to denote the time discretization parameter, and the set of time intervals $\mathcal{M}_{\tau}:=\left\{I_{1}, \ldots, I_{N}\right\}$ is called the time mesh.

We approximate the exact solution $u: \bar{I} \rightarrow V$ by means of a function $u_{\tau}: \bar{I} \rightarrow V$ which is piecewise a polynomial of some order $k \geq 0$ with respect to time. For $B \in\{L, V\}$, let $\mathbb{P}_{k}\left(I_{n}, B\right):=\left\{w: I_{n} \rightarrow B: w(t)=\sum_{j=0}^{k} W^{j} t^{j}, \forall t \in I_{n}, W^{j} \in B, \forall j\right\}$ be the space of $B$-valued polynomials in time of order $k$ over $I_{n}$ and let

$$
X_{\tau}^{k}(B):=\left\{w_{\tau}: \bar{I} \rightarrow B:\left.w_{\tau}\right|_{I_{n}} \in \mathbb{P}_{k}\left(I_{n}, B\right) \quad \forall I_{n} \in \mathcal{M}_{\tau}\right\} .
$$

Then, we seek the time semi-discrete solution $u_{\tau}$ in the space $X_{\tau}^{k}(V)$. It is possible to consider a polynomial degree $k_{n}$ specific to each time interval $I_{n}$. All what follows extends to this more general setting.

Let $w_{\tau}$ be a function in $X_{\tau}^{k}(B)$. Then, $w_{\tau}$ can be discontinuous at the discrete times $t_{n}$, for all $n=0, \ldots, N$, while $w_{\tau}$ is by definition continuous from the left at $t_{n}$, for all $n=1, \ldots, N$, i.e., $w_{\tau}\left(t_{n}\right)=\lim _{t \uparrow t_{n}} w_{\tau}(t)$. Moreover, $w_{\tau}(0)$ has to be specified separately since $0 \notin I_{1}$. The space $X_{\tau}^{k}(B)$ is a subspace of

$$
C^{-1}\left(\mathcal{M}_{\tau}, B\right):=\left\{w_{\tau}: \bar{I} \rightarrow B:\left.w_{\tau}\right|_{I_{n}} \in C^{0}\left(I_{n}, B\right), w_{\tau}\left(t_{n-1}^{+}\right) \text {exists } \forall I_{n} \in \mathcal{M}_{\tau}\right\},
$$

with the notation $w_{\tau}\left(t_{n-1}^{+}\right):=\lim _{t \downarrow t_{n-1}} w_{\tau}(t)$. Functions in $C^{-1}\left(\mathcal{M}_{\tau}, B\right)$ are by definition continuous from the left at all $t_{n}, n=1, \ldots, N$, and their value at 0 has to be specified separately. For all $w_{\tau} \in C^{-1}\left(\mathcal{M}_{\tau}, B\right)$, the jump of $w_{\tau}$ at $t_{n}$, for all $n=0, \ldots, N-1$, is defined as

$$
\left[w_{\tau}\right]_{n}:=w_{\tau}\left(t_{n}^{+}\right)-w_{\tau}\left(t_{n}\right) .
$$


The discontinuous Galerkin method of order $k$ (in short, $\mathrm{dG}(k)$ ) applied as time semidiscretization of problem (4) reads: Find $u_{\tau} \in X_{\tau}^{k}(V)$ such that $u_{\tau}(0)=u_{0}$ and

$$
\sum_{n=1}^{N} \int_{I_{n}}\left(\partial_{t} u_{\tau}+A u_{\tau}, v_{\tau}\right)_{L} d t+\sum_{n=0}^{N-1}\left(\left[u_{\tau}\right]_{n}, v_{\tau}\left(t_{n}^{+}\right)\right)_{L}=\int_{I}\left(f, v_{\tau}\right)_{L} d t \quad \forall v_{\tau} \in Y_{\tau}^{k}(L),
$$

with test space $Y_{\tau}^{k}(L):=\left\{v: I \rightarrow L:\left.v\right|_{I_{n}} \in \mathbb{P}_{k}\left(I_{n}, L\right) \quad \forall I_{n} \in \mathcal{M}_{\tau}\right\}$.

Problem (8) can be decoupled into a sequence of local problems by choosing test functions supported on a single time interval $I_{n}$. Then, the time semi-discrete solution $u_{\tau}$ can be determined by successively solving a local problem on $I_{n}$. Using the known value $u_{\tau}\left(t_{n-1}\right)$ from the previous time interval (and $u_{0}$ for $n=1$ ), the local problem on $I_{n}$ reads: Find $\left.u_{\tau}\right|_{I_{n}} \in \mathbb{P}_{k}\left(I_{n}, V\right)$ such that

$$
\int_{I_{n}}\left(\partial_{t} u_{\tau}+A u_{\tau}, v_{\tau}\right)_{L} d t+\left(\left[u_{\tau}\right]_{n-1}, v_{\tau}\left(t_{n-1}^{+}\right)\right)_{L}=\int_{I_{n}}\left(f, v_{\tau}\right)_{L} d t \quad \forall v_{\tau} \in \mathbb{P}_{k}\left(I_{n}, L\right) .
$$

In practice, the right-hand side of (9) is evaluated by means of some numerical integration formula. In the context of the $\mathrm{dG}(k)$-method in time, a natural choice is to consider the $(k+1)$ point right-sided Gauss-Radau quadrature formula on each time interval $I_{n}$. For a function $g \in C^{-1}\left(\mathcal{M}_{\tau}, \mathbb{R}\right)$ and $I_{n} \in \mathcal{M}_{\tau}$, this formula takes the form

$$
Q_{n}(g):=\frac{\tau_{n}}{2} \sum_{\mu=1}^{k+1} \widehat{w}_{\mu} g\left(t_{n, \mu}\right) \approx \int_{I_{n}} g(t) d t
$$

where $t_{n, \mu} \in I_{n}$ are the integration points and $\widehat{w}_{\mu}>0$ the weights. Note that $t_{n, k+1}=t_{n}$ so that $g\left(t_{n, k+1}\right)$ is equal to $g\left(t_{n}\right)$ (recall that $g$ is continuous from the left at $t_{n}$ ). It is known that (10) is exact for all polynomials in $\mathbb{P}_{2 k}\left(I_{n}\right)$. Then, using (10) on the right-hand side of (9) leads to the numerically-integrated version of (9):

$$
\int_{I_{n}}\left(\partial_{t} u_{\tau}+A u_{\tau}, v_{\tau}\right)_{L} d t+\left(\left[u_{\tau}\right]_{n-1}, v_{\tau}\left(t_{n-1}^{+}\right)\right)_{L}=Q_{n}\left(\left(f, v_{\tau}\right)_{L}\right) \quad \forall v_{\tau} \in \mathbb{P}_{k}\left(I_{n}, L\right) .
$$

To rewrite (11), we define the Lagrange interpolation operator $I_{\mathcal{\tau}}^{\mathrm{GR}}: C^{0}(\bar{I}, L) \rightarrow X_{\mathcal{\tau}}^{k}(L)$ by means of the conditions

$$
I_{\tau}^{\mathrm{GR}} w\left(t_{n, \mu}\right)=w\left(t_{n, \mu}\right), \quad \mu=1, \ldots, k+1, n=1, \ldots, N, \quad I_{\tau}^{\mathrm{GR}} w(0)=w(0),
$$

using the $(k+1)$ right-sided Gauss-Radau integration points $t_{n, \mu} \in I_{n}$. Then, (11) is equivalent to

$$
\int_{I_{n}}\left(\partial_{t} u_{\tau}+A u_{\tau}, v_{\tau}\right)_{L} d t+\left(\left[u_{\tau}\right]_{n-1}, v_{\tau}\left(t_{n-1}^{+}\right)\right)_{L}=\int_{I_{n}}\left(I_{\tau}^{\mathrm{GR}} f, v_{\tau}\right)_{L} d t \quad \forall v_{\tau} \in \mathbb{P}_{k}\left(I_{n}, L\right)
$$

Let us briefly look, for example, at the $\mathrm{dG}(1)$-method. Here, we apply the 2-point rightsided Gauss-Radau quadrature formula with points $t_{n, 1}=t_{n-1}+\tau_{n} / 3, t_{n, 2}=t_{n}$ and reference weights $\hat{w}_{1}=3 / 2, \hat{w}_{2}=1 / 2$. On the time interval $I_{n}$, we have to solve for the two unknowns $U_{n}^{j}=u_{\tau}\left(t_{n, j}\right)$ for $j=1,2$. The coupled $(2 \times 2)$-block-system for $U_{n}^{1}, U_{n}^{2} \in V$ reads:

$$
\begin{array}{lll}
\frac{3}{4} U_{n}^{1}+\frac{\tau_{n}}{2} A U_{n}^{1} & +\frac{1}{4} U_{n}^{2} & =u_{\tau}\left(t_{n-1}\right)+\frac{\tau_{n}}{2} f\left(t_{n, 1}\right), \\
-\frac{9}{4} U_{n}^{1} & +\frac{5}{4} U_{n}^{2}+\frac{\tau_{n}}{2} A U_{n}^{2} & =-u_{\tau}\left(t_{n-1}\right)+\frac{\tau_{n}}{2} f\left(t_{n, 2}\right) .
\end{array}
$$




\section{$3.2 \quad$ A lifting operator}

As a key point of our analysis, we introduce the lifting operator

$$
\mathcal{L}_{\tau}: X_{\tau}^{k}(B) \rightarrow X_{\tau}^{k+1}(B) \cap C^{0}(\bar{I}, B),
$$

such that, for all $n=1, \ldots, N$,

$$
\mathcal{L}_{\tau} w_{\tau}(t):=w_{\tau}(t)-\left[w_{\tau}\right]_{n-1} \vartheta_{n}(t) \quad \forall t \in I_{n}=\left(t_{n-1}, t_{n}\right],
$$

and $\mathcal{L}_{\tau} w_{\tau}(0)=w_{\tau}(0)$. Here, $\vartheta_{n} \in \mathbb{P}_{k+1}\left(I_{n}, \mathbb{R}\right)$ is defined by means of the integration points $t_{n, \mu}$ of the $(k+1)$-point right-sided Gauss-Radau quadrature formula on the interval $I_{n}$ as

$$
\vartheta_{n}(t):=\prod_{\mu=1}^{k+1} \frac{t-t_{n, \mu}}{t_{n-1}-t_{n, \mu}} \quad \forall t \in I_{n} .
$$

The continuity in time of $\mathcal{L}_{\tau} w_{\tau}$ follows from the properties $\vartheta_{n}\left(t_{n-1}^{+}\right)=1$ and $\vartheta_{n}\left(t_{n}\right)=$ $\vartheta_{n}\left(t_{n, k+1}\right)=0$ together with the definition of the jump $\left[w_{\tau}\right]_{n-1}$. Since $\vartheta_{n}(t)$ vanishes at the integration points, we get the property

$$
\mathcal{L}_{\tau} w_{\tau}\left(t_{n, \mu}\right):=w_{\tau}\left(t_{n, \mu}\right) \quad \forall \mu=1, \ldots, k+1, \forall n=1, \ldots, N .
$$

The lifting operator $\mathcal{L}_{\tau}$ can be more generally defined for functions in $C^{-1}\left(\mathcal{M}_{\tau}, B\right)$ and then maps onto $C^{0}(\bar{I}, B)$.

\subsection{Space discretization by stabilized FEM}

In this section, we briefly recall some basic elements on the discretization of the space differential operator $A$ by means of a stabilized FEM. For clarity, we consider functions depending only on the space variable, and return to the full space-time setting in Section 3.4.

Let $\mathcal{T}_{h}$ be a shape-regular mesh of $\Omega$ and let $V_{h}$ be a finite element space built on that mesh, where $h$ denotes the mesh-size. For simplicity, we assume that the mesh $\mathcal{T}_{h}$ is affine and that $\Omega$ has a polygonal $(d=2)$ or polyhedral $(d=3)$ boundary. To fix the ideas, we assume that $V_{h}$ contains at least piecewise polynomials of order $r$, yielding the following local approximation property: For all $w \in H^{r+1}(\Omega)$, there exists an interpolate $i_{h} w \in V_{h}$ such that, for $m \in\{0,1\}$,

$$
\left|w-i_{h} w\right|_{H^{m}(T)} \lesssim h_{T}^{r+1-m}|w|_{H^{r+1}\left(\Delta_{T}\right)} \quad \forall T \in \mathcal{T}_{h},
$$

where $h_{T}$ stands for the diameter of $T$ and $\Delta_{T}$ is a set of mesh elements neighboring $T$.

The differential operator $A: V \rightarrow L$ is approximated by a discrete differential operator $A_{h}: W+V_{h} \rightarrow V_{h}$, where $W$ is a dense subspace of $V$ used to assert the consistency of $A_{h}$ (see (20) below); typically, $W=H^{s}(\Omega), s \geq 1$. For the operator $A_{h}$, we require the following properties:

- Consistency: Letting $P_{h}: L \rightarrow V_{h}$ denote the $L^{2}$-orthogonal projector onto $V_{h}$,

$$
A_{h} w=P_{h} A w \quad \forall w \in W .
$$


- Discrete coercivity and boundedness on orthogonal subscales:

$$
\begin{array}{rlrl}
\left\|v_{h}\right\|^{2} & \lesssim\left(A_{h} v_{h}, v_{h}\right)_{L} & & \forall v_{h} \in V_{h}, \\
\left(A_{h}\left(w-P_{h} w\right), v_{h}\right)_{L} \lesssim\left\|w-P_{h} w\right\|_{h, \frac{1}{2}}\left\|v_{h}\right\| & & \forall v_{h} \in V_{h}, w \in W,
\end{array}
$$

where $\|\cdot\|$ and $\|\cdot\|_{h, \frac{1}{2}}$ are mesh-dependent norms on $\left(W+V_{h}\right)$ satisfying

$$
\mu_{0}^{1 / 2}\|v\|_{L} \leq\|v\| \leq\|v\|_{h, \frac{1}{2}} \quad \forall v \in\left(W+V_{h}\right) .
$$

- Discrete inf-sup stability: For all $v_{h} \in V_{h}$, there exists $w_{h} \in V_{h}$ such that

$$
\left\|v_{h}\right\|_{\sharp}^{2} \lesssim\left(A_{h} v_{h}, w_{h}\right)_{L} \quad \text { and } \quad\left\|w_{h}\right\|_{\sharp} \leq\left\|v_{h}\right\|_{\sharp},
$$

where $\|\cdot\|_{\sharp}$ is a mesh-dependent norm on $V_{h}$ such that $\left\|v_{h}\right\| \leq\left\|v_{h}\right\|_{\sharp}$ for all $v_{h} \in V_{h}$, and $\|w\|_{\sharp} \lesssim\|w\|_{W}$ for all $w \in W$. In what follows, $\|\cdot\|_{\sharp}$ is termed the discrete graph norm since it provides a control on the advective derivative.

We now present two examples for the discrete operator $A_{h}$ matching the above framework, one obtained using the Continuous Interior Penalty (CIP) method based on $H^{1}$-conforming finite elements and gradient jump penalty at interfaces and the other obtained using the $\mathrm{dG}$ method (in space) with upwind fluxes. Some slight adaptations of the consistency assumption are needed to handle other stablizations for $H^{1}$-conforming finite elements (e.g., local projection, subgrid viscosity, orthogonal subscales). Mesh faces are collected in the set $\mathcal{F}_{h}$ split into the set of interior faces, $\mathcal{F}_{h}^{\text {int }}$, and boundary faces, $\mathcal{F}_{h}^{\text {ext }}$. For $F \in \mathcal{F}_{h}^{\text {int }}$, there are $T^{-}, T^{+}$in $\mathcal{T}_{h}$ such that $F=\partial T^{-} \cap \partial T^{+}, n_{F}$ is the unit normal to $F$ pointing from $T^{-}$to $T^{+}$, and for a piecewise smooth enough function $v$, we define its jump and mean value at $F$ as $[v]:=\left.v\right|_{T^{-}}-\left.v\right|_{T^{+}}$and $\{\{v\}\}:=\frac{1}{2}\left(\left.v\right|_{T^{-}}+\left.v\right|_{T^{+}}\right)$, respectively. The arbitrariness in the sign of $[v]$ is irrelevant. Meshes can possess hanging nodes when using $\mathrm{dG}$ methods under the usual assumption that face diameters are comparable to local element diameters. In what follows, $h_{F}$ denotes the diameter of $F \in \mathcal{F}_{h}$, and for $R \in\{T, \partial T, F\},(\cdot, \cdot)_{L, R}$ the $L^{2}(R)$-inner product with associated norm $\|\cdot\|_{L, R}$.

In the CIP method, see $[6,9,7]$, the discrete space $V_{h}$ is $H^{1}$-conforming, and, letting $W=H^{2}(\Omega)$, the discrete operator $A_{h}^{\text {cip }}$ is given, for all $v \in W+V_{h}, w_{h} \in V_{h}$, by

$$
\begin{aligned}
\left(A_{h}^{\text {cip }} v, w_{h}\right)_{L}= & \left(\sigma v+\beta \cdot \nabla v, w_{h}\right)_{L}+\sum_{F \in \mathcal{F}_{h}^{\text {ext }}}\left(\left(\beta \cdot n_{F}\right)^{\ominus} v, w_{h}\right)_{L, F} \\
& +\sum_{F \in \mathcal{F}_{h}^{\text {int }}}\left(\gamma_{F}[\nabla v],\left[\nabla w_{h}\right]\right)_{L, F},
\end{aligned}
$$

where $x^{\ominus}:=\frac{1}{2}(|x|-x)$ denotes the negative part of a real number $x$ and $\gamma_{F}=\varrho h_{F}^{2}\left|\beta \cdot n_{F}\right|$ with $\varrho>0$ a user-dependent parameter. Then, consistency holds. Discrete coercivity holds with the mesh-dependent norm on $\left(W+V_{h}\right)$

$$
\|v\|^{2}:=\mu_{0}\|v\|_{L}^{2}+\sum_{F \in \mathcal{F}_{h}^{\text {ext }}}\left\|\left|\beta \cdot n_{F}\right|^{1 / 2} v\right\|_{L, F}^{2}+\sum_{F \in \mathcal{F}_{h}^{\text {int }}}\left\|\gamma_{F}^{1 / 2}[\nabla v]\right\|_{L, F}^{2},
$$


boundedness on orthogonal subscales with $\|v\|_{h, \frac{1}{2}}^{2}=\|v\|^{2}+\sum_{T \in \mathcal{T}_{h}}\left\{h_{T}^{-1}\|v\|_{L, T}^{2}+\|v\|_{L, \partial T}^{2}\right\}$, and discrete inf-sup stability with $\left\|v_{h}\right\|_{\sharp}^{2}:=\left\|v_{h}\right\|^{2}+\sum_{T \in \mathcal{T}_{h}} h_{T}\left\|\beta \cdot \nabla v_{h}\right\|_{L, T}^{2}$.

In the DG method, see $[30,29,5,19,16]$, the discrete space $V_{h}$ is spanned by piecewise polynomials with no continuity explicitly enforced at interfaces, and, letting $W=H^{1}(\Omega)$, the discrete operator $A_{h}^{\mathrm{dg}}$ is given, for all $v \in W+V_{h}, w_{h} \in V_{h}$, by

$$
\begin{aligned}
\left(A_{h}^{\mathrm{dg}} v, w_{h}\right)_{L}= & \sum_{T \in \mathcal{T}_{h}}\left(\sigma v+\beta \cdot \nabla v, w_{h}\right)_{L, T}+\sum_{F \in \mathcal{F}_{h}^{\text {ext }}}\left(\left(\beta \cdot n_{F}\right)^{\ominus} v, w_{h}\right)_{L, F} \\
& -\sum_{F \in \mathcal{F}_{h}^{\text {int }}}\left(\left(\beta \cdot n_{F}\right)[v],\left\{\left\{w_{h}\right\}\right\}\right)_{L, F}+\sum_{F \in \mathcal{F}_{h}^{\text {int }}}\left(\gamma_{F}[v],\left[w_{h}\right]\right)_{L, F},
\end{aligned}
$$

where $\gamma_{F}=\varrho\left|\beta \cdot n_{F}\right|$ with $\varrho>0$ a user-dependent parameter $\left(\varrho=\frac{1}{2}\right.$ yields the classical upwind fluxes). Then, consistency holds. Discrete coercivity holds with the mesh-dependent norm on $\left(W+V_{h}\right)$

$$
\|v\|^{2}:=\mu_{0}\|v\|_{L}^{2}+\sum_{F \in \mathcal{F}_{h}^{\text {ext }}}\left\|\left|\beta \cdot n_{F}\right|^{1 / 2} v\right\|_{L, F}^{2}+\sum_{F \in \mathcal{F}_{h}^{\text {int }}}\left\|\gamma_{F}^{1 / 2}[v]\right\|_{L, F}^{2},
$$

boundedness on orthogonal subscales with $\|v\|_{h, \frac{1}{2}}^{2}=\|v\|^{2}+\sum_{T \in \mathcal{T}_{h}}\|v\|_{L, \partial T}^{2}$, and discrete infsup stability with $\left\|v_{h}\right\|_{\sharp}^{2}:=\left\|v_{h}\right\|^{2}+\sum_{T \in \mathcal{T}_{h}} h_{T}\left\|\beta \cdot \nabla v_{h}\right\|_{L, T}^{2}$.

To derive error estimates, we need suitable approximation properties of the $L^{2}$-orthogonal projector $P_{h}$, namely

$$
\left\|w-P_{h} w\right\|_{h, \frac{1}{2}}+\left\|w-P_{h} w\right\|_{\sharp} \lesssim h^{r+1 / 2}|w|_{H^{r+1}(\Omega)} .
$$

Such a property is satisfied for shape-regular meshes and any polynomial degree $r$ for the DG method since the projector $P_{h}$ enjoys local approximation properties. For conforming FEM with CIP, we use the recent result of [3] focusing for simplicity on simplicial meshes. We assume that to each mesh element $T \in \mathcal{T}_{h}$, we can assign a nonnegative integer $k_{T}$, the level of $T$, such that $h_{T} \sim 2^{-k_{T}}$ and such that for any two elements sharing a vertex, their level differs at most by one. We refer to [3] for a discussion on the relevance of this hypothesis in the context of adaptive and more general nonuniform meshes. Following [3], we also assume that, for $d=2, r \leq 12$ and, for $d=3, r \leq 7$. In what follows, the above assumptions are referred to as $\mathbf{( H )}$.

Lemma 1 Under Assumption (H), the approximation property (29) holds.

Proof. We only prove the bound on the $\|\cdot\|_{h, \frac{1}{2}}$-norm; the proof for the other bound is similar. By the triangle inequality, we infer that

$$
\left\|w-P_{h} w\right\|_{h, \frac{1}{2}} \leq\left\|w-i_{h} w\right\|_{h, \frac{1}{2}}+\left\|P_{h}\left(w-i_{h} w\right)\right\|_{h, \frac{1}{2}}
$$

since $i_{h} v \in V_{h}$. The first term on the right-hand side is bounded by $h^{r+1 / 2}|w|_{H^{r+1}(\Omega)}$ owing to (19). Concerning the second term, we first observe using inverse and trace inequalities that $\left\|v_{h}\right\|_{h, \frac{1}{2}} \lesssim\left\|\mathfrak{h}^{-1 / 2} v_{h}\right\|_{L}$ for all $v_{h} \in V_{h}$, where $\mathfrak{h}$ denotes the piecewise constant function 
equal locally to the mesh element diameter. Moreover, the Cauchy-Schwarz inequality implies $\left\|\mathfrak{h}^{-1 / 2} v_{h}\right\|_{L} \leq\left\|\mathfrak{h}^{-1} v_{h}\right\|_{L}^{1 / 2}\left\|v_{h}\right\|_{L}^{1 / 2}$. Since Lemma 4.1 in [3] shows that $\left\|\mathfrak{h}^{-1} P_{h} v\right\|_{L} \lesssim\left\|\mathfrak{h}^{-1} v\right\|_{L}$ for all $v \in L$, we infer with $v=w-i_{h} w$ and $v_{h}=P_{h} v$ that

$$
\left\|P_{h}\left(w-i_{h} w\right)\right\|_{h, \frac{1}{2}} \lesssim\left\|\mathfrak{h}^{-1}\left(w-i_{h} w\right)\right\|_{L}^{1 / 2}\left\|w-i_{h} w\right\|_{L}^{1 / 2},
$$

whence the assertion results from (19).

\subsection{Full space-time discretization}

In the full space-time discretization, we approximate on each time interval $I_{n}=\left(t_{n-1}, t_{n}\right]$ the time semi-discrete solution $u_{\tau}$ by means of a fully discrete solution $u_{\tau h}$ using a finite element space $V_{h}^{n} \subset L$ resulting from a mesh $\mathcal{T}_{h}^{n}$ which can change from one time interval to the next. The corresponding discrete differential operator is $A_{h}^{n}: W+V_{h}^{n} \rightarrow V_{h}^{n}$ and satisfies the design conditions of Section 3.3 uniformly in $n$. Concerning mesh-dependent norms like $\|\cdot\| \|$, we use a subscript $n$ as in $\|\cdot\|_{n}$ to indicate that this norm is defined using the mesh $\mathcal{T}_{h}^{n}$. The global solution space for the fully discrete solution $u_{\tau h}$ is

$$
X_{\tau h}^{k}:=\left\{v_{\tau h}: \bar{I} \rightarrow L ;\left.v_{\tau h}\right|_{I_{n}} \in \mathbb{P}_{k}\left(I_{n}, V_{h}^{n}\right) \quad \forall I_{n} \in \mathcal{M}_{\tau}\right\} .
$$

Let $u_{0 h} \in V_{h}^{0}$ be an approximation of the initial condition $u_{0}$. The initial mesh $\mathcal{T}_{h}^{0}$ used to build $V_{h}^{0}$ can differ from the mesh $\mathcal{T}_{h}^{1}$ used in the first time interval $I_{1}$.

For brevity, we only present the fully discrete problem on each time interval, i.e., resulting from the space discretization of (11). For all $n=1, \ldots, N$, using the known value $u_{\tau h}\left(t_{n-1}\right) \in$ $V_{h}^{n-1}$ from the previous time interval (and $u_{0 h}$ for $n=1$ ), the local, fully discrete problem on $I_{n}$ reads: Find $\left.u_{\tau h}\right|_{I_{n}} \in \mathbb{P}_{k}\left(I_{n}, V_{h}^{n}\right)$ such that, for all $v_{\tau h} \in \mathbb{P}_{k}\left(I_{n}, V_{h}^{n}\right)$,

$$
\int_{I_{n}}\left(\partial_{t} u_{\tau h}+A_{h}^{n} u_{\tau h}, v_{\tau h}\right)_{L} d t+\left(\left[u_{\tau h}\right]_{n-1}, v_{\tau h}\left(t_{n-1}^{+}\right)\right)_{L}=Q_{n}\left(\left(f, v_{\tau h}\right)_{L}\right) .
$$

We now derive a useful result allowing us to rewrite the fully discrete scheme (31) using the lifting operator $\mathcal{L}_{\tau}$ introduced in Section 3.2.

Lemma 2 For all $n=1, \ldots, N$, (31) is equivalent to

$$
\int_{I_{n}}\left(\partial_{t} \mathcal{L}_{\tau} u_{\tau h}+A_{h}^{n} u_{\tau h}, v_{\tau h}\right)_{L} d t=Q_{n}\left(\left(f, v_{\tau h}\right)_{L}\right) \quad \forall v_{\tau h} \in \mathbb{P}_{k}\left(I_{n}, V_{h}^{n}\right)
$$

Proof. For all $n=1, \ldots, N$, using integration by parts for the $\vartheta_{n}$-term, we obtain

$$
\begin{aligned}
\int_{I_{n}}\left(\partial_{t} \mathcal{L}_{\tau} u_{\tau h}, v_{\tau h}\right)_{L} d t= & \int_{I_{n}}\left(\partial_{t} u_{\tau h}, v_{\tau h}\right)_{L} d t+\int_{I_{n}}\left(\left[u_{\tau h}\right]_{n-1} \vartheta_{n}, \partial_{t} v_{\tau h}\right)_{L} d t \\
& +\left(\left[u_{\tau h}\right]_{n-1}, v_{\tau h}\left(t_{n-1}^{+}\right)\right)_{L},
\end{aligned}
$$

since $\vartheta_{n}\left(t_{n-1}\right)=1$ and $\vartheta_{n}\left(t_{n}\right)=0$. The integrand of the second integral on the right-hand side is in $\mathbb{P}_{2 k}\left(I_{n}, \mathbb{R}\right)$. Then, the $(k+1)$-point right-sided Gauss-Radau quadrature formula is exact and the integral vanishes. 


\section{Preparation for the error analysis}

\subsection{Basic stability result}

To state the consistency properties of the fully discrete problem, it is convenient to define, in each time interval $I_{n}, n=1, \ldots, N$, the bilinear form

$$
\widetilde{B}_{h}^{n}(w, v):=Q_{n}\left(\left(\partial_{t} w, v\right)_{L}\right)+Q_{n}\left(\left(A_{h}^{n} w, v\right)_{L}\right),
$$

where $v \in C^{-1}\left(\mathcal{M}_{\tau}, L\right)$ and $w$ must satisfy the following smoothness conditions, expressed as $w \in \tilde{X}$ with

$$
\tilde{X}:=\left\{w: \bar{I} \rightarrow L ; w\left(t_{n, \mu}\right) \in W+V_{h}^{n},\left.\partial_{t} w\right|_{I_{n}}\left(t_{n, \mu}\right) \in L, \forall \mu=1, \ldots, k+1, n=1, \ldots, N\right\},
$$

where $t_{n, \mu} \in I_{n}$ are the right-sided Gauss-Radau integration points. Note that, although the function $w=\mathcal{L}_{\tau} u_{\tau h}$ restricted to $I_{n}$ is in $\mathbb{P}_{k+1}\left(I_{n}, V_{h}^{n-1}+V_{h}^{n}\right)$, this function satisfies $w\left(t_{n, \mu}\right) \in V_{h}^{n}$ owing to (18); hence, $w \in \tilde{X}$. The bilinear form $\widetilde{B}_{h}^{n}$ is closely related to the fully discrete problem, as we now show.

Lemma 3 The fully discrete solution $u_{\tau h} \in X_{\tau h}^{k}$ is such that, for all $n=1, \ldots, N$,

$$
\widetilde{B}_{h}^{n}\left(\mathcal{L}_{\tau} u_{\tau h}, v_{\tau h}\right)=Q_{n}\left(\left(f, v_{\tau h}\right)_{L}\right) \quad \forall v_{\tau h} \in \mathbb{P}_{k}\left(I_{n}, V_{h}^{n}\right) .
$$

Proof. Using definition (33) yields

$$
\widetilde{B}_{h}^{n}\left(\mathcal{L}_{\tau} u_{\tau h}, v_{\tau h}\right)=Q_{n}\left(\left(\partial_{t} \mathcal{L}_{\tau} u_{\tau h}, v_{\tau h}\right)_{L}\right)+Q_{n}\left(\left(A_{h}^{n} \mathcal{L}_{\tau} u_{\tau h}, v_{\tau h}\right)_{L}\right) .
$$

Since $\left(\partial_{t} \mathcal{L}_{\tau} u_{\tau h}, v_{\tau h}\right)_{L}$ is in $\mathbb{P}_{2 k}\left(I_{n}\right)$, we obtain $Q_{n}\left(\left(\partial_{t} \mathcal{L}_{\tau} u_{\tau h}, v_{\tau h}\right)_{L}\right)=\int_{I_{n}}\left(\partial_{t} \mathcal{L}_{\tau} u_{\tau h}, v_{\tau h}\right)_{L} d t$. Moreover, using $(18)$ and since $\left(A_{h}^{n} u_{\tau h}, v_{\tau h}\right)_{L}$ is in $\mathbb{P}_{2 k}\left(I_{n}, \mathbb{R}\right)$, we infer that

$$
Q_{n}\left(\left(A_{h}^{n} \mathcal{L}_{\tau} u_{\tau h}, v_{\tau h}\right)_{L}\right)=Q_{n}\left(\left(A_{h}^{n} u_{\tau h}, v_{\tau h}\right)_{L}\right)=\int_{I_{n}}\left(A_{h}^{n} u_{\tau h}, v_{\tau h}\right)_{L} d t
$$

We conclude using (32).

The discrete bilinear form $\widetilde{B}_{h}^{n}$ satisfies a basic stability result which is the starting point of our error analysis.

Lemma 4 (Stability) For given $w \in C^{0}(\bar{I}, L) \cap X_{\tau}^{k+1}(L) \cap \tilde{X}$, let $I_{\tau}^{\mathrm{GR}} w \in X_{\tau}^{k}(L)$ be defined in (12). Then, for all $n=1, \ldots, N$, the following bound holds:

$$
\widetilde{B}_{h}^{n}\left(w, I_{\tau}^{\mathrm{GR}} w\right) \geq \frac{1}{2}\left\|w\left(t_{n}\right)\right\|_{L}^{2}-\frac{1}{2}\left\|w\left(t_{n-1}\right)\right\|_{L}^{2}+\frac{1}{2}\left\|\left[I_{\tau}^{\mathrm{GR}} w\right]_{n-1}\right\|_{L}^{2}+\alpha Q_{n}\left(\|w\|_{n}^{2}\right) .
$$

Proof. Using definition (33) yields

$$
\widetilde{B}_{h}^{n}\left(w, I_{\tau}^{\mathrm{GR}} w\right)=Q_{n}\left(\left(\partial_{t} w, I_{\tau}^{\mathrm{GR}} w\right)_{L}\right)+Q_{n}\left(\left(A_{h}^{n} w, I_{\tau}^{\mathrm{GR}} w\right)_{L}\right) .
$$


Concerning the first term on the right-hand side, since the integrand is in $\mathbb{P}_{2 k}\left(I_{n}, \mathbb{R}\right)$, we obtain $Q_{n}\left(\left(\partial_{t} w, I_{\tau}^{\mathrm{GR}} w\right)_{L}\right)=\int_{I_{n}}\left(\partial_{t} w, I_{\tau}^{\mathrm{GR}} w\right)_{L} d t$. Moreover, since $w \in \mathbb{P}_{k+1}\left(I_{n}, L\right)$, we observe that $w(t)=I_{\tau}^{\mathrm{GR}} w(t)+d_{n-1} \vartheta_{n}(t) \quad$ with $\quad d_{n-1}:=w\left(t_{n-1}\right)-I_{\tau}^{\mathrm{GR}} w\left(t_{n-1}^{+}\right)=-\left[I_{\tau}^{\mathrm{GR}} w\right]_{n-1}$,

$t \in I_{n}$ and $\vartheta_{n}$ defined in (17). This implies

$$
\begin{aligned}
Q_{n}\left(\left(\partial_{t} w, I_{\tau}^{\mathrm{GR}} w\right)_{L}\right) & =\int_{I_{n}}\left(\partial_{t} w, w-d_{n-1} \vartheta_{n}\right)_{L} d t \\
& =\int_{I_{n}}\left(\partial_{t} w, w\right)_{L} d t-\int_{I_{n}}\left(\partial_{t} w, d_{n-1} \vartheta_{n}\right)_{L} d t \\
& =\int_{I_{n}} \frac{1}{2} \frac{d}{d t}\|w\|_{L}^{2} d t-\int_{I_{n}}\left(\partial_{t} I_{\tau}^{\mathrm{GR}} w+d_{n-1} \partial_{t} \vartheta_{n}, d_{n-1} \vartheta_{n}\right)_{L} d t \\
& =\int_{I_{n}} \frac{1}{2} \frac{d}{d t}\|w\|_{L}^{2} d t-\left\|d_{n-1}\right\|_{L}^{2} \int_{I_{n}} \frac{1}{2} \frac{d}{d t}\left|\vartheta_{n}\right|^{2} d t
\end{aligned}
$$

since $\int_{I_{n}}\left(\partial_{t} I_{\tau}^{\mathrm{GR}} w, d_{n-1} \vartheta_{n}\right)_{L} d t=0$ being the integrand in $\mathbb{P}_{2 k}\left(I_{n}, \mathbb{R}\right)$ and vanishing at all right-sided Gauss-Radau integration points in $I_{n}$. As a result,

$$
Q_{n}\left(\left(\partial_{t} w, I_{\tau}^{\mathrm{GR}} w\right)_{L}\right)=\frac{1}{2}\left\|w\left(t_{n}\right)\right\|_{L}^{2}-\frac{1}{2}\left\|w\left(t_{n-1}\right)\right\|_{L}^{2}+\frac{1}{2}\left\|d_{n-1}\right\|_{L}^{2} .
$$

Furthermore, using (21) (discrete coercivity) and since $w$ and $I_{\tau}^{\mathrm{GR}} w$ coincide at all $t_{n, \mu}$, $\mu=1, \ldots, k+1$, we infer that

$$
Q_{n}\left(\left(A_{h}^{n} w, I_{\tau}^{\mathrm{GR}} w\right)_{L}\right)=Q_{n}\left(\left(A_{h}^{n} I_{\tau}^{\mathrm{GR}} w, I_{\tau}^{\mathrm{GR}} w\right)_{L}\right) \geq \alpha Q_{n}\left(\left\|I_{\tau}^{\mathrm{GR}} w\right\|_{n}^{2}\right)=\alpha Q_{n}\left(\|w\|_{n}^{2}\right),
$$

whence the assertion follows.

\subsection{Construction of a special interpolate in time}

In this section, we assume $k \geq 1$. Let $B$ be a Banach space, typically $B \in\{L, V\}$. For a function $u \in C^{1}(\bar{I}, B)$, we define a time-polynomial interpolate $R_{\tau}^{k+1} u \in C^{0}(\bar{I}, B)$ whose restriction to $I_{n}=\left(t_{n-1}, t_{n}\right]$ is in $\mathbb{P}_{k+1}\left(I_{n}, B\right)$. We first choose a Lagrange/Hermite interpolate $I_{\tau}^{k+2} u \in C^{0}(\bar{I}, B)$ such that, for all $n=1, \ldots, N,\left.I_{\tau}^{k+2} u\right|_{I_{n}} \in \mathbb{P}_{k+2}\left(I_{n}, B\right)$ and

$$
I_{\tau}^{k+2} u\left(t_{n}\right)=u\left(t_{n}\right) \quad \text { and } \quad \partial_{t} I_{\tau}^{k+2} u\left(t_{n}\right)=\partial_{t} u\left(t_{n}\right) \quad \forall n=0, \ldots, N .
$$

For $k=1$, these conditions fully determine $I_{\tau}^{k+2} u$ (for $k=0$, the above construction is not possible), while, for $k \geq 2$, values at, say, additional Lagrange nodes can be prescribed inside each $I_{n}$ so that, assuming $u$ smooth enough,

$$
\begin{aligned}
\left\|\partial_{t} u-\partial_{t} I_{\tau}^{k+2} u\right\|_{C^{0}\left(\bar{I}_{n}, B\right)} & \lesssim \tau_{n}^{k+2}|u|_{C^{k+3}\left(\bar{I}_{n}, B\right)}, \\
\left\|\partial_{t}^{2} u-\partial_{t}^{2} I_{\tau}^{k+2} u\right\|_{C^{0}\left(\bar{I}_{n}, B\right)} & \lesssim \tau_{n}^{k+1}|u|_{C^{k+3}\left(\bar{I}_{n}, B\right)} .
\end{aligned}
$$

Then, we define $\left.R_{\tau}^{k+1} u\right|_{I_{n}} \in \mathbb{P}_{k+1}\left(I_{n}, B\right)$ by means of the $(k+2)$ conditions

$$
\begin{aligned}
\partial_{t} R_{\tau}^{k+1} u\left(t_{n, \mu}\right) & =\partial_{t} I_{\tau}^{k+2} u\left(t_{n, \mu}\right) \quad \forall \mu=1, \ldots, k+1, \\
R_{\tau}^{k+1} u\left(t_{n-1}^{+}\right) & =I_{\tau}^{k+2} u\left(t_{n-1}\right),
\end{aligned}
$$

and finally we set $R_{\tau}^{k+1} u(0)=u(0)=u_{0}$. 
Lemma 5 Assume $k \geq 1$. The function $R_{\tau}^{k+1} u$ is continuous in time on $\bar{I}$ with $R_{\tau}^{k+1} u\left(t_{n}\right)=$ $u\left(t_{n}\right)$ for all $n=0, \ldots, N$.

Proof. The function $R_{\tau}^{k+1} u$ is continuous at 0 since $R_{\tau}^{k+1} u\left(0^{+}\right)=I_{\tau}^{k+2} u(0)=u(0)=$ $R_{\tau}^{k+1} u(0)$. Let now $n=1, \ldots, N$. From (39) and (40), we obtain for an arbitrary timeindependent test function $v \in L$,

$$
\begin{aligned}
\left(R_{\tau}^{k+1} u\left(t_{n}\right), v\right)_{L} & =\left(R_{\tau}^{k+1} u\left(t_{n-1}^{+}\right), v\right)_{L}+\int_{I_{n}}\left(\partial_{t} R_{\tau}^{k+1} u, v\right)_{L} d t \\
& =\left(I_{\tau}^{k+2} u\left(t_{n-1}\right), v\right)_{L}+Q_{n}\left(\left(\partial_{t} R_{\tau}^{k+1} u, v\right)_{L}\right) \\
& =\left(I_{\tau}^{k+2} u\left(t_{n-1}\right), v\right)_{L}+Q_{n}\left(\left(\partial_{t} I_{\tau}^{k+2} u, v\right)_{L}\right) .
\end{aligned}
$$

Since $\partial_{t} I_{\tau}^{k+2} u$ is in $\mathbb{P}_{k+1}\left(I_{n}, B\right)$ and $k+1 \leq 2 k$ for all $k \geq 1$, we obtain $Q_{n}\left(\left(\partial_{t} I_{\tau}^{k+2} u, v\right)_{L}\right)=$ $\int_{I_{n}}\left(\partial_{t} I_{\tau}^{k+2} u, v\right)_{L} d t$. As a result,

$$
\left(R_{\tau}^{k+1} u\left(t_{n}\right), v\right)_{L}=\left(I_{\tau}^{k+2} u\left(t_{n-1}\right), v\right)_{L}+\int_{I_{n}}\left(\partial_{t} I_{\tau}^{k+2} u, v\right)_{L} d t=\left(I_{\tau}^{k+2} u\left(t_{n}\right), v\right)_{L},
$$

proving the assertion.

Lemma 6 Assume $k \geq 1$. For all $n=1, \ldots, N$ and all $u \in C^{k+2}\left(\bar{I}_{n}, B\right)$, the following bound holds:

$$
\left\|u-R_{\tau}^{k+1} u\right\|_{C^{0}\left(\bar{I}_{n}, B\right)} \lesssim \tau_{n}^{k+2}|u|_{C^{k+2}\left(\bar{I}_{n}, B\right)} .
$$

Moreover, the bound $\left\|R_{\tau}^{k+1} u\right\|_{C^{0}\left(\bar{I}_{n}, B\right)} \lesssim \max \left(1, \tau_{n}\right)\|u\|_{C^{1}\left(\bar{I}_{n}, B\right)}$ holds for all $u \in C^{1}\left(\bar{I}_{n}, B\right)$.

Proof. See appendix.

Corollary 7 Assume $k \geq 1$. For all $n=1, \ldots, N$ and all $u \in C^{k+2}\left(\bar{I}_{n}, B\right)$, the following bound holds:

$$
\left\|\partial_{t} u-\partial_{t} R_{\tau}^{k+1} u\right\|_{C^{0}\left(\bar{I}_{n}, B\right)} \lesssim \tau_{n}^{k+1}|u|_{C^{k+2}\left(\bar{I}_{n}, B\right)} .
$$

Moreover, the bound $\left\|\partial_{t} R_{\tau}^{k+1} u\right\|_{C^{0}\left(\bar{I}_{n}, B\right)} \lesssim\|u\|_{C^{1}\left(\bar{I}_{n}, B\right)}$ holds for all $u \in C^{1}\left(\bar{I}_{n}, B\right)$.

Proof. Let $L_{\tau}^{k+1} u$ be the Lagrange interpolate of $u$ in $\mathbb{P}_{k+1}\left(\bar{I}_{n}, B\right)$ based on the $(k+1)$ right-sided Gauss-Radau integration points on each $\bar{I}_{n}$ and the left endpoint $t_{n-1}$. Then,

$$
\begin{aligned}
\left\|\partial_{t} u-\partial_{t} R_{\tau}^{k+1} u\right\|_{C^{0}\left(\bar{I}_{n}, B\right)} & \leq\left\|\partial_{t} u-\partial_{t} L_{\tau}^{k+1} u\right\|_{C^{0}\left(\bar{I}_{n}, B\right)}+\left\|\partial_{t} L_{\tau}^{k+1} u-\partial_{t} R_{\tau}^{k+1} u\right\|_{C^{0}\left(\bar{I}_{n}, B\right)} \\
& \lesssim \tau_{n}^{k+1}|u|_{C^{k+2}\left(\bar{I}_{n}, B\right)}+\tau_{n}^{-1}\left\|L_{\tau}^{k+1} u-R_{\tau}^{k+1} u\right\|_{C^{0}\left(\bar{I}_{n}, B\right)},
\end{aligned}
$$

and using the triangle inequality, we infer that

$$
\left\|L_{\tau}^{k+1} u-R_{\tau}^{k+1} u\right\|_{C^{0}\left(\bar{I}_{n}, B\right)} \leq\left\|L_{\mathcal{\tau}}^{k+1} u-u\right\|_{C^{0}\left(\bar{I}_{n}, B\right)}+\left\|u-R_{\tau}^{k+1} u\right\|_{C^{0}\left(\bar{I}_{n}, B\right)} \lesssim \tau_{n}^{k+2}|u|_{C^{k+2}\left(\bar{I}_{n}, B\right)},
$$


thereby proving (42). The proof of the stability bound is similar upon observing that $\left\|\partial_{t} R_{\tau}^{k+1} u\right\|_{C^{0}\left(\bar{I}_{n}, B\right)} \lesssim \tau_{n}^{-1}\left\|R_{\tau}^{k+1} u-u\left(t_{n}\right)\right\|_{C^{0}\left(\bar{I}_{n}, B\right)} \leq \tau_{n}^{-1}\left\|R_{\tau}^{k+1} u-u\right\|_{C^{0}\left(\bar{I}_{n}, B\right)}+\tau_{n}^{-1}\left\|u-u\left(t_{n}\right)\right\|_{C^{0}\left(\bar{I}_{n}, B\right)}$. The first term on the right-hand side is bounded by $\|u\|_{C^{1}\left(\bar{I}_{n}, B\right)}$ (see the proof of Lemma 6 in the Appendix using a constant Taylor polynomial) and the second one by $|u|_{C^{1}\left(\bar{I}_{n}, B\right)}$. This completes the proof.

\section{$5 \quad L^{2}$-norm error estimates}

This section is devoted to the $L^{\infty}\left(L^{2}\right)$ and $L^{2}\left(L^{2}\right)$ error estimates, first for static and then for time-varying meshes. Our main goal is to estimate the error defined as

$$
\tilde{e}(t):=u(t)-\mathcal{L}_{\tau} u_{\tau h}(t) \quad \forall t \in \bar{I} .
$$

We observe that the error is evaluated using the post-processed solution $\mathcal{L}_{\tau} u_{\tau h}$ and that $\tilde{e}$ is continuous in time on $\bar{I}$ and, moreover, $\tilde{e} \in \tilde{X}$, see (34), if we assume for our analysis that the exact solution $u$ has at least the regularity $u \in C^{0}(\bar{I}, W) \cap C^{1}(\bar{I}, L)$. Throughout this section, we assume $k \geq 1$. The case $k=0$ corresponding to the implicit Euler scheme is briefly discussed in Remark 15. We start with the following consistency result.

Lemma 8 (Consistency) Assume $u \in C^{0}(\bar{I}, W) \cap C^{1}(\bar{I}, L)$. For all $n=1, \ldots, N$, the following equality holds:

$$
\widetilde{B}_{h}^{n}\left(\tilde{e}, v_{\tau h}\right)=0 \quad \forall v_{\tau h} \in X_{\tau h}^{k}
$$

Proof. We recall from Lemma 3 that, for all $n=1, \ldots, N$ and all $v_{\tau h} \in \mathbb{P}_{k}\left(I_{n}, V_{h}\right)$, $\widetilde{B}_{h}^{n}\left(\mathcal{L}_{\tau} u_{\tau h}, v_{\tau h}\right)=Q_{n}\left(\left(f, v_{\tau h}\right)_{L}\right)$. Moreover, since the exact solution satisfies $\partial_{t} u\left(t_{n, \mu}\right)+$ $A u\left(t_{n, \mu}\right)=f\left(t_{n, \mu}\right)$ for all $\mu=1, \ldots, k+1$, we infer using the consistency of $A_{h}$, see (20), that

$$
\widetilde{B}_{h}^{n}\left(u, v_{\tau h}\right)=Q_{n}\left(\left(\partial_{t} u+A_{h} u, v_{\tau h}\right)_{L}\right)=Q_{n}\left(\left(\partial_{t} u+A u, v_{\tau h}\right)_{L}\right)=Q_{n}\left(\left(f, v_{\tau h}\right)_{L}\right),
$$

whence the assertion follows.

\subsection{Static meshes}

In the case of static meshes, we drop the superscript $n$ on the mesh $\mathcal{T}_{h}$, the finite element space $V_{h}$, and the discrete differential operator $A_{h}$. Our analysis hinges on the following error decomposition:

$$
\tilde{e}(t)=\underbrace{\left(u(t)-P_{h} R_{\tau}^{k+1} u(t)\right)}_{=: \eta(t)}+\underbrace{\left(P_{h} R_{\tau}^{k+1} u(t)-\mathcal{L}_{\tau} u_{\tau h}(t)\right)}_{=\tilde{e}_{\tau h}(t)} \quad \forall t \in \bar{I},
$$

observing that both $\eta$ and $\tilde{e}_{\tau h}$ are continuous in time on $\bar{I}$. The function $\eta$ is referred to as the interpolation error. Note that both $\eta$ as well as $\tilde{e}_{\tau h}$ are in the space $\tilde{X}$, see (34), so that they can be used as arguments in the bilinear form $\widetilde{B}_{h}^{n}$. 
Lemma 9 (Boundedness) For all $n=1, \ldots, N$, the following bound holds:

$$
\left|\widetilde{B}_{h}^{n}\left(\eta, v_{\tau h}\right)\right| \lesssim\left\{\left(E_{n}^{\mathrm{T}}(u)\right)^{2}+\left(E_{n}^{\mathrm{S}}(u)\right)^{2}\right\}^{1 / 2}\left\{Q_{n}\left(\left\|v_{\tau h}\right\|^{2}\right)\right\}^{1 / 2},
$$

with the time and space errors respectively given by

$$
\begin{aligned}
& \left(E_{n}^{\mathrm{T}}(u)\right)^{2}=Q_{n}\left(\left\|\partial_{t}\left(u-I_{\tau}^{k+2} u\right)\right\|_{L}^{2}+\left\|u-R_{\tau}^{k+1} u\right\|_{V}^{2}\right), \\
& \left(E_{n}^{\mathrm{S}}(u)\right)^{2}=Q_{n}\left(\left\|R_{\tau}^{k+1} u-P_{h} R_{\tau}^{k+1} u\right\|_{h, \frac{1}{2}}^{2}\right) .
\end{aligned}
$$

Proof. We decompose $\widetilde{B}_{h}^{n}\left(\eta, v_{\tau h}\right)$ as

$$
\begin{aligned}
\widetilde{B}_{h}^{n}\left(\eta, v_{\tau h}\right)= & \left.Q_{n}\left(\partial_{t}\left(u-P_{h} R_{\tau}^{k+1} u\right), v_{\tau h}\right)_{L}\right)+Q_{n}\left(\left(A_{h}\left(u-R_{\tau}^{k+1} u\right), v_{\tau h}\right)_{L}\right) \\
& +Q_{n}\left(\left(A_{h}\left(R_{\tau}^{k+1} u-P_{h} R_{\tau}^{k+1} u\right), v_{\tau h}\right)_{L}\right)=: T_{1}+T_{2}+T_{3} .
\end{aligned}
$$

Concerning $T_{1}$, we can drop the projection $P_{h}$ and use the property (39) of $R_{\tau}^{k+1}$ followed by the Cauchy-Schwarz inequality to infer that

$$
\left|T_{1}\right| \leq\left\{Q_{n}\left(\left\|\partial_{t}\left(u-I_{\tau}^{k+2} u\right)\right\|_{L}^{2}\right)\right\}^{1 / 2}\left\{Q_{n}\left(\left\|v_{\tau h}\right\|_{L}^{2}\right)\right\}^{1 / 2}
$$

Concerning $T_{2}$, since $\left(u-R_{\tau}^{k+1} u\right)$ is in $W$, the consistency of the discrete operator $A_{h}$ together with the Cauchy-Schwarz inequality and the boundedness of $A$ lead to

$$
\begin{aligned}
\left|T_{2}\right| & =\left|Q_{n}\left(\left(A\left(u-R_{\tau}^{k+1} u\right), v_{\tau h}\right)_{L}\right)\right| \\
& \lesssim\left\{Q_{n}\left(\left\|u-R_{\tau}^{k+1} u\right\|_{V}^{2}\right)\right\}^{1 / 2}\left\{Q_{n}\left(\left\|v_{\tau h}\right\|_{L}^{2}\right)\right\}^{1 / 2} .
\end{aligned}
$$

Concerning $T_{3}$, we use boundedness on orthogonal subscales, see (22), to infer that

$$
\left|T_{3}\right| \lesssim\left\{Q_{n}\left(\left\|R_{\tau}^{k+1} u-P_{h} R_{\tau}^{k+1} u\right\|_{h, \frac{1}{2}}^{2}\right)\right\}^{1 / 2}\left\{Q_{n}\left(\left\|v_{\tau h}\right\|^{2}\right)\right\}^{1 / 2}
$$

Collecting the above bounds yields the assertion since $\|\cdot\|_{L} \lesssim\|\cdot\|$, see (23).

Lemma 10 (Estimates on $\left.\tilde{e}_{\tau h}\right)$ For all $m=1, \ldots, N$, the following bound holds:

$$
\left\|\tilde{e}_{\tau h}\left(t_{m}\right)\right\|_{L}^{2}+\sum_{n=1}^{m} Q_{n}\left(\left\|\tilde{e}_{\tau h}\right\|^{2}\right) \lesssim\left(E_{0}\right)^{2}+\sum_{n=1}^{m}\left\{\left(E_{n}^{\mathrm{T}}(u)\right)^{2}+\left(E_{n}^{\mathrm{S}}(u)\right)^{2}\right\},
$$

with initial error $E_{0}=\left\|P_{h} u_{0}-u_{0 h}\right\|_{L}$. Moreover, assuming $\tau_{n} \lesssim \tau_{n-1}$ for all $n=2, \ldots, N$ and $\tau_{1} \lesssim 1$, the following bound holds:

$$
\left\|\tilde{e}_{\tau h}\right\|_{L^{2}(I, L)}^{2} \lesssim\left(E_{0}\right)^{2}+\sum_{n=1}^{N}\left\{\left(E_{n}^{\mathrm{T}}(u)\right)^{2}+\left(E_{n}^{\mathrm{S}}(u)\right)^{2}\right\} .
$$


Proof. Owing to Lemma 8 (consistency) and the error decomposition (45), we infer that $\widetilde{B}_{h}^{n}\left(\tilde{e}_{\tau h}, v_{\tau h}\right)=-\widetilde{B}_{h}^{n}\left(\eta, v_{\tau h}\right)$, so that using Lemma 9 leads to

$$
\widetilde{B}_{h}^{n}\left(\tilde{e}_{\tau h}, v_{\tau h}\right) \lesssim\left\{\left(E_{n}^{\mathrm{T}}(u)\right)^{2}+\left(E_{n}^{\mathrm{S}}(u)\right)^{2}\right\}^{1 / 2}\left\{Q_{n}\left(\left\|v_{\tau h}\right\|^{2}\right)\right\}^{1 / 2}
$$

Setting $v_{\tau h}=I_{\tau}^{\mathrm{GR}} \tilde{e}_{\tau h}$ and using the stability property of $\widetilde{B}_{h}^{n}$ stated in Lemma 4 for $w=\tilde{e}_{\tau h}$ together with the continuity in time of $\tilde{e}_{\tau h}$ and a Young inequality, we infer that

$$
\frac{1}{2}\left\|\tilde{e}_{\tau h}\left(t_{n}\right)\right\|_{L}^{2}-\frac{1}{2}\left\|\tilde{e}_{\tau h}\left(t_{n-1}\right)\right\|_{L}^{2}+Q_{n}\left(\left\|\tilde{e}_{\tau h}\right\|^{2}\right) \lesssim\left(E_{n}^{\mathrm{T}}(u)\right)^{2}+\left(E_{n}^{\mathrm{S}}(u)\right)^{2},
$$

where we have dropped the nonnegative jump term at $t_{n-1}$ from the stability property. Taking an arbitrary $m=1, \ldots, N$ and summing the above inequality from $n=1$ to $m$ leads to (49) since $\tilde{e}_{\tau h}(0)=P_{h} u_{0}-u_{0 h}$. To prove $(50)$, we start with the estimate

$$
\left\|w_{\tau}\right\|_{L^{2}\left(I_{n}, L\right)}^{2} \lesssim Q_{n}\left(\left\|w_{\tau}\right\|_{L}^{2}\right)+\tau_{n}\left\|w_{\tau}\left(t_{n-1}^{+}\right)\right\|_{L}^{2} \quad \forall w_{\tau} \in \mathbb{P}_{k+1}\left(I_{n}, L\right),
$$

which follows by transformation from $\bar{I}_{n}$ to the reference element $\hat{I}=[-1,1]$ and application of a norm equivalence on the time polynomial space $\mathbb{P}_{k+1}(\hat{I}, L)$. Applying this inequality to $\tilde{e}_{\tau h}$ which is continuous in time leads to

$$
\left\|\tilde{e}_{\tau h}\right\|_{L^{2}\left(I_{n}, L\right)}^{2} \lesssim Q_{n}\left(\left\|\tilde{e}_{\tau h}\right\|_{L}^{2}\right)+\tau_{n}\left\|\tilde{e}_{\tau h}\left(t_{n-1}\right)\right\|_{L}^{2}
$$

Summing this bound from $n=1$ to $N$ and observing that, for all $n \geq 2, \tau_{n}\left\|\tilde{e}_{\tau h}\left(t_{n-1}\right)\right\|_{L}^{2} \lesssim$ $\tau_{n-1}\left\|\tilde{e}_{\tau h}\left(t_{n-1}\right)\right\|_{L}^{2} \lesssim Q_{n-1}\left(\left\|\tilde{e}_{\tau h}\right\|_{L}^{2}\right)$ since $\tau_{n} \lesssim \tau_{n-1}$ and $t_{n-1, k+1}=t_{n-1}$, we infer that

$$
\left\|\tilde{e}_{\tau h}\right\|_{L^{2}(I, L)}^{2} \lesssim \sum_{n=1}^{N} Q_{n}\left(\left\|\tilde{e}_{\tau h}\right\|_{L}^{2}\right)+\tau_{1}\left\|\tilde{e}_{\tau h}(0)\right\|_{L}^{2},
$$

whence (50) follows since $\tau_{1} \lesssim 1$.

Theorem 11 ( $L^{2}$-error estimate) Let $u$ be the exact solution and let $u_{\tau h}$ be the fully discrete solution. Assume $k \geq 1$ and $\tau_{n} \lesssim 1$ for all $n=1, \ldots, N$. Assume $(\boldsymbol{H})$ in the case of conforming FEM. Then, for the error $\tilde{e}(t)$ defined in (43), the following bound holds for all $m=1, \ldots, N$

$$
\left\|\tilde{e}\left(t_{m}\right)\right\|_{L}^{2} \lesssim\left(E_{0}\right)^{2}+t_{m} \max _{1 \leq n \leq m}\left\{C_{n}^{\mathrm{T}}(u) \tau_{n}^{2(k+2)}+C_{n}^{\mathrm{S}}(u) h^{2 r+1}\right\}+C_{m}^{\prime}(u) h^{2(r+1)}
$$

with $C_{n}^{\mathrm{T}}(u):=|u|_{C^{k+3}\left(\bar{I}_{n}, L\right)}^{2}+|u|_{C^{k+2}\left(\bar{I}_{n}, V\right)}^{2}, C_{n}^{\mathrm{S}}(u):=\|u\|_{C^{1}\left(\bar{I}_{n}, H^{r+1}(\Omega)\right)}^{2}$, and $C_{m}^{\prime}(u)=\left|u\left(t_{m}\right)\right|_{H^{r+1}(\Omega)}^{2}$. Moreover, assuming $\tau_{n} \lesssim \tau_{n-1}$ for all $n=2, \ldots, N$, the following bound holds:

$$
\|\tilde{e}\|_{L^{2}(I, L)}^{2} \lesssim\left(E_{0}\right)^{2}+T \max _{1 \leq n \leq N}\left\{C_{n}^{\mathrm{T}}(u) \tau_{n}^{2(k+2)}+C_{n}^{\mathrm{S}}(u) h^{2 r+1}\right\}
$$

Proof. From definitions (47)-(48) of $E_{n}^{\mathrm{T}}(u)$ and $E_{n}^{\mathrm{S}}(u)$ for all $n=1, \ldots, N$, we infer that

$$
\begin{aligned}
\left(E_{n}^{\mathrm{T}}(u)\right)^{2} & \lesssim \tau_{n}\left(\tau_{n}^{2(k+2)}|u|_{C^{k+3}\left(\bar{I}_{n}, L\right)}^{2}+\tau_{n}^{2(k+2)}|u|_{C^{k+2}\left(\bar{I}_{n}, V\right)}^{2}\right) \\
\left(E_{n}^{\mathrm{S}}(u)\right)^{2} & \lesssim \tau_{n} h^{2 r+1}\|u\|_{C^{1}\left(\bar{I}_{n}, H^{r+1}(\Omega)\right)}^{2}
\end{aligned}
$$


where the bound on $E_{n}^{\mathrm{T}}(u)$ results from (37) with $B=L$ and (41) with $B=V$, and that on $E_{n}^{\mathrm{S}}(u)$ from the approximation property $(29)$ of $P_{h}$ combined with the stability of $R_{\tau}^{k+1}$ from Lemma 6 with $B=H^{r+1}(\Omega)$ and the assumption $\tau_{n} \lesssim 1$. Moreover, recalling the error decomposition (45), we observe that, for all $n=1, \ldots, N$,

$$
\left\|\eta\left(t_{n}\right)\right\|_{L}=\left\|u\left(t_{n}\right)-P_{h} R_{\tau}^{k+1} u\left(t_{n}\right)\right\|_{L}=\left\|u\left(t_{n}\right)-P_{h} u\left(t_{n}\right)\right\|_{L} \lesssim h^{r+1}\left|u\left(t_{n}\right)\right|_{H^{r+1}(\Omega)},
$$

where we used Lemma 5 , and

$$
\begin{aligned}
\|\eta\|_{L^{2}\left(I_{n}, L\right)}^{2} & =\left\|u-P_{h} R_{\tau}^{k+1} u\right\|_{L^{2}\left(I_{n}, L\right)}^{2} \leq 2\left\|u-P_{h} u\right\|_{L^{2}\left(I_{n}, L\right)}^{2}+2\left\|P_{h}\left(u-R_{\tau}^{k+1} u\right)\right\|_{L^{2}\left(I_{n}, L\right)}^{2} \\
& \leq 2\left\|u-P_{h} u\right\|_{L^{2}\left(I_{n}, L\right)}^{2}+2\left\|u-R_{\tau}^{k+1} u\right\|_{L^{2}\left(I_{n}, L\right)}^{2} \\
& \lesssim \tau_{n}\left(h^{2(r+1)}\|u\|_{C^{0}\left(\bar{I}_{n}, H^{r+1}(\Omega)\right)}^{2}+\tau_{n}^{2(k+2)}|u|_{C^{k+2}\left(\bar{I}_{n}, L\right)}^{2}\right) .
\end{aligned}
$$

We conclude using Lemma 10 and the triangle inequality, as well as $h \leq \operatorname{diam}(\Omega) \lesssim 1$ and $\|\cdot\|_{L} \lesssim\|\cdot\|_{V}$ for $(52)$.

Remark 12 (Assumption on the time steps) The assumption $\tau_{n} \lesssim \tau_{n-1}$ is quite mild; it means that the time step can be increased at most by a uniformly bounded factor. The assumption $\tau_{n} \lesssim 1$ is also quite mild; it means that the time steps resolve the fastest time scale present in the governing equations which is here given by $\min \left(\mu_{0}, L_{\beta}\right)$ with $\mu_{0}$ from (3) and $L_{\beta}$ the Lipschitz constant of $\beta$.

Remark 13 (Initial error) The initial error $E_{0}$ vanishes when the discrete initial condition is chosen to be $u_{0 h}=P_{h} u_{0}$. Otherwise, this error is typically of order $h^{r+1}$ if $u_{0}$ is smooth enough.

Remark 14 (Estimate on the error $\left(u-u_{\tau h}\right)$ ) Since $\tilde{e}\left(t_{m}\right)=u\left(t_{m}\right)-\mathcal{L}_{\tau} u_{\tau h}\left(t_{m}\right)=u\left(t_{m}\right)-$ $u_{\tau h}\left(t_{m}\right)$ for all $m=1, \ldots, N$, the right-hand side of (51) also bounds the error $\| u\left(t_{m}\right)$ $u_{\tau h}\left(t_{m}\right) \|_{L}$, showing that a superconvergent $L^{2}$-error estimate of order $\left(\tau^{k+2}+h^{r+1 / 2}\right)$ also holds under the assumptions of Theorem 11 for the original fully discrete solution $u_{\tau}$ at the discrete nodes defining the time partition. Instead, the bound on $\left\|u-u_{\tau h}\right\|_{L^{2}(I, L)}$ is of order $\left(\tau^{k+1}+h^{r+1 / 2}\right)$ which is optimal with respect to $\tau$. This bound can be derived from

$$
\left\|I_{\tau}^{\mathrm{GR}} u-u_{\tau h}\right\|_{L^{2}\left(I_{n}, L\right)}^{2}=Q_{n}\left(\left\|I_{\tau}^{\mathrm{GR}} u-u_{\tau h}\right\|_{L}^{2}\right)=Q_{n}\left(\|\tilde{e}\|_{L}^{2}\right) \lesssim Q_{n}\left(\left\|\tilde{e}_{\tau h}\right\|_{L}^{2}\right)+Q_{n}\left(\|\eta\|_{L}^{2}\right),
$$

where $\tilde{e}, \tilde{e}_{\tau h}$, and $\eta$ are defined in (45). The sum over all $n=1, \ldots, N$ yields as in the previous analysis the same upper bound as the right-hand side of (52). Then, invoking the triangle inequality $\left\|u-u_{\tau h}\right\|_{L^{2}(I, L)} \leq\left\|u-I_{\tau}^{\mathrm{GR}} u\right\|_{L^{2}(I, L)}+\left\|I_{\tau}^{\mathrm{GR}} u-u_{\tau h}\right\|_{L^{2}(I, L)}$ yields the claim. Finally, since $\left[u_{\tau h}\right]_{n-1} \vartheta_{n}(t)=u_{\tau h}(t)-\mathcal{L}_{\tau} u_{\tau h}(t)$ for all $t \in I_{n}$, we infer by means of the triangle inequality the same bound for the jump seminorm $\left(\sum_{n=1}^{N}\left\|\left[u_{\tau h}\right]_{n-1}\right\|_{L}^{2}\right)^{1 / 2}$.

Remark 15 (Implicit Euler) For $k=0$, the interpolate $R_{\tau}^{k+1} u$ is not available since the construction of Section 4.2 requires $k \geq 1$. The analysis proceeds by replacing in the above proofs $R_{\tau}^{k+1} u$ by the piecewise affine Lagrange interpolate of $u$ in time and leads to an error bound of order $\left(\tau+h^{r+1 / 2}\right)$ for the error $\tilde{e}$ in the $L$ norm at the discrete times defining the time partition and in the $L^{2}(I, L)$ norm. 


\subsection{Time-varying meshes}

Now, we allow that, on each time interval $I_{n}=\left(t_{n-1}, t_{n}\right]$, we can have a new mesh $\mathcal{T}_{h}^{n}$ which can be created from the previous mesh $\mathcal{T}_{h}^{n-1}$ by means of local refinements and derefinements. Therefore, it is necessary to use the superscript $n$ also for the finite element space $V_{h}^{n}$ and the discrete differential operator $A_{h}^{n}$. By $h_{n}$ we denote the maximum of all diameters $h_{K}$ of the mesh cells $K \in \mathcal{T}_{h}^{n}$. For each $n=0,1, \ldots, N$, let $P_{h}^{n}: L \rightarrow V_{h}^{n}$ denote the $L^{2}$-projector onto $V_{h}^{n}$. For a time-dependent function $w: \bar{I} \rightarrow L$, we define its space projection $P_{h} w: \bar{I} \rightarrow L$ as

$$
\left(P_{h} w\right)(t):=\left\{\begin{array}{lll}
P_{h}^{n}(w(t)) & \text { if } t \in I_{n}=\left(t_{n-1}, t_{n}\right] \\
P_{h}^{0}(w(0)) & \text { if } t=0 .
\end{array}\right.
$$

Note that, even for a continuous function $w \in C^{0}(\bar{I}, L)$, its space projection $P_{h} w$ can be discontinuous in time at the discrete points $t_{n-1}$, i.e., $P_{h} w \in C^{-1}\left(\mathcal{M}_{\tau}, L\right)$ with the jump

$$
\left[P_{h} w\right]_{n-1}=P_{h}^{n} w\left(t_{n-1}^{+}\right)-P_{h}^{n-1} w\left(t_{n-1}\right) \in V_{h}^{n}+V_{h}^{n-1},
$$

which is in general non-zero if $V_{h}^{n} \neq V_{h}^{n-1}$.

\subsubsection{Error estimates with projection error}

Recall the error $\tilde{e}(t)$ defined in (43). In the case of time-varying meshes, the decomposition (45) of the error $\tilde{e}(t)$ has to be modified as

$$
\tilde{e}(t)=\underbrace{\left(u(t)-\mathcal{L}_{\tau} P_{h} R_{\tau}^{k+1} u(t)\right)}_{=: \eta(t)}+\underbrace{\left(\mathcal{L}_{\tau} P_{h} R_{\tau}^{k+1} u(t)-\mathcal{L}_{\tau} u_{\tau h}(t)\right)}_{=\tilde{e}_{\tau h}(t)} \quad \forall t \in \bar{I},
$$

where the use of the lifting operator in the definition of $\eta$ allows us to recover a continuous function in time. We can write $\eta(t)$ for $t \in I_{n}$ as

$$
\eta(t)=\underbrace{\left(u(t)-P_{h}^{n} R_{\tau}^{k+1} u(t)\right)}_{=: \eta^{\text {old }}(t)}+\left[P_{h} u\right]_{n-1} \vartheta_{n}(t),
$$

where we have used that $R_{\tau}^{k+1} u\left(t_{n-1}\right)=u\left(t_{n-1}\right)$ and $\eta^{\text {old }}(t)$ denotes the interpolation error used for the $L^{2}$-analysis in the case of static meshes. The part $\left[P_{h} u\right]_{n-1}$ leads to an extra term in the error analysis for time-varying meshes and can be regarded as the projection error for the time interval $I_{n}$. Note that again $\eta$ and $\tilde{e}_{\tau h}$ are contained in the space $\tilde{X}$ defined in (34). Let $\Pi_{h}^{n-1}: V_{h}^{n-1}+V_{h}^{n} \rightarrow V_{h}^{n-1}$ denote an $L^{2}$-stable, linear quasi-interpolation operator satisfying the following properties:

$$
\begin{array}{rlrl}
\Pi_{h}^{n-1} v_{h} & =v_{h} & & \forall v_{h} \in V_{h}^{n-1}, \\
\left\|\Pi_{h}^{n-1} v_{h}\right\|_{L} \lesssim\left\|v_{h}\right\|_{L} & \forall v_{h} \in V_{h}^{n-1}+V_{h}^{n} .
\end{array}
$$

Lemma 16 (Boundedness) For all $n=1, \ldots, N$, the following bound holds:

$$
\left|\widetilde{B}_{h}^{n}\left(\eta, v_{\tau h}\right)\right| \lesssim\left\{\left(E_{n}^{\mathrm{T}}(u)\right)^{2}+\left(E_{n}^{\mathrm{S}}(u)\right)^{2}\right\}^{1 / 2}\left\{Q_{n}\left(\left\|v_{\tau h}\right\|_{n}^{2}\right)\right\}^{1 / 2}+E_{n}^{\mathrm{P}}(u)\left\|\left[v_{\tau h}\right]_{n-1}\right\|_{L},
$$


with $E_{n}^{\mathrm{T}}(u)$ given by $(47),\left(E_{n}^{\mathrm{S}}(u)\right)^{2}:=Q_{n}\left(\left\|R_{\tau}^{k+1} u-P_{h}^{n} R_{\tau}^{k+1} u\right\|_{h, \frac{1}{2}, n}^{2}\right)$, and the local projection error defined by

$$
E_{n}^{\mathrm{P}}(u):=\sup _{v_{h} \in V_{h}^{n}} \frac{\left(u\left(t_{n-1}\right)-P_{h}^{n-1} u\left(t_{n-1}\right), v_{h}-\Pi_{h}^{n-1} v_{h}\right)_{L}}{\left\|v_{h}-\Pi_{h}^{n-1} v_{h}\right\|_{L}},
$$

with the convention that the ratio is zero if $v_{h} \in V_{h}^{n-1}$, which means, in particular, that $E_{n}^{\mathrm{P}}(u)=0$ in the case that $V_{h}^{n} \subset V_{h}^{n-1}$.

Proof. Since $\vartheta_{n}$ vanishes at the $(k+1)$ right-sided Gauss-Radau integration points, we can decompose $\widetilde{B}_{h}^{n}\left(\eta, v_{\tau h}\right)$ as

$$
\begin{aligned}
\widetilde{B}_{h}^{n}\left(\eta, v_{\tau h}\right) & =\widetilde{B}_{h}^{n}\left(\eta^{\text {old }}, v_{\tau h}\right)+\widetilde{B}_{h}^{n}\left(\left[P_{h} u\right]_{n-1} \vartheta_{n}, v_{\tau h}\right) \\
& =\left(T_{1}+T_{2}+T_{3}\right)+\underbrace{Q_{n}\left(\left(\left[P_{h} u\right]_{n-1} \vartheta_{n}^{\prime}, v_{\tau h}\right)_{L}\right)}_{=: T_{4}},
\end{aligned}
$$

where $T_{1,2,3}$ denote the terms introduced in the proof of Lemma 9. Concerning $T_{4}$, we exploit that the integrand is a polynomial in $\mathbb{P}_{2 k}\left(I_{n}, \mathbb{R}\right)$ to infer that

$$
\begin{aligned}
T_{4} & =\int_{I_{n}}\left(\left[P_{h} u\right]_{n-1} \vartheta_{n}^{\prime}, v_{\tau h}\right)_{L} d t=-\int_{I_{n}}\left(\left[P_{h} u\right]_{n-1} \vartheta_{n}, \partial_{t} v_{\tau h}\right)_{L} d t-\left(\left[P_{h} u\right]_{n-1}, v_{\tau h}\left(t_{n-1}^{+}\right)\right)_{L} \\
& =\left(\left[u-P_{h} u\right]_{n-1}, v_{\tau h}\left(t_{n-1}^{+}\right)\right)_{L} \\
& =-\left(u\left(t_{n-1}\right)-P_{h}^{n-1} u\left(t_{n-1}\right), v_{\tau h}\left(t_{n-1}^{+}\right)\right)_{L} \\
& =-\left(u\left(t_{n-1}\right)-P_{h}^{n-1} u\left(t_{n-1}\right),\left[v_{\tau h}\right]_{n-1}\right)_{L}
\end{aligned}
$$

using that $\vartheta_{n}\left(t_{n, \mu}\right)=0$ for $\mu=1, \ldots, k+1, \vartheta_{n}\left(t_{n-1}^{+}\right)=1,[u]_{n-1}=0, v_{\tau h}\left(t_{n-1}^{+}\right) \in V_{h}^{n}$, and $v_{\tau h}\left(t_{n-1}\right) \in V_{h}^{n-1}$. We use the notation $v_{h}^{+}:=v_{\tau h}\left(t_{n-1}^{+}\right) \in V_{h}^{n}$ and $v_{h}^{n-1}:=v_{\tau h}\left(t_{n-1}\right) \in V_{h}^{n-1}$. If $v_{h}^{+}$turns out to be in $V_{h}^{n-1}$, then $T_{4}=0$ which obviously satisfies $\left|T_{4}\right| \leq E_{n}^{\mathrm{P}}(u)\left\|\left[v_{\tau h}\right]_{n-1}\right\|_{L}$. Otherwise, $v_{h}^{+}-\Pi_{h}^{n-1} v_{h}^{+} \neq 0$ and since, owing to (56),

$$
\left[v_{\tau h}\right]_{n-1}-\Pi_{h}^{n-1}\left[v_{\tau h}\right]_{n-1}=\left(v_{h}^{+}-\Pi_{h}^{n-1} v_{h}^{+}\right)-\left(v_{h}^{n-1}-\Pi_{h}^{n-1} v_{h}^{n-1}\right)=v_{h}^{+}-\Pi_{h}^{n-1} v_{h}^{+},
$$

we infer that

$$
\begin{aligned}
\left|T_{4}\right| & =\left|\left(u\left(t_{n-1}\right)-P_{h}^{n-1} u\left(t_{n-1}\right), v_{h}^{+}-\Pi_{h}^{n-1} v_{h}^{+}\right)_{L}\right| \\
& =\frac{\left|\left(u\left(t_{n-1}\right)-P_{h}^{n-1} u\left(t_{n-1}\right), v_{h}^{+}-\Pi_{h}^{n-1} v_{h}^{+}\right)_{L}\right|}{\left\|v_{h}^{+}-\Pi_{h}^{n-1} v_{h}^{+}\right\|_{L}}\left\|\left[v_{\tau h}\right]_{n-1}-\Pi_{h}^{n-1}\left[v_{\tau h}\right]_{n-1}\right\|_{L} \\
& \lesssim E_{n}^{\mathrm{P}}(u)\left\|\left[v_{\tau h}\right]_{n-1}\right\|_{L},
\end{aligned}
$$

where we used (57) in the last bound. Collecting the bounds for $T_{1,2,3}$ from the proof of Lemma 9 and the above bound for $T_{4}$ yields the assertion.

Theorem 17 ( $L^{2}$-error estimate) Let $u$ be the exact solution and let $u_{\tau h} \in X_{\tau h}^{k}$ be the fully discrete solution. Assume $k \geq 1$ and $\tau_{n} \lesssim 1$ for all $n=1, \ldots, N$. Assume $(\boldsymbol{H})$ in the 
case of conforming FEM. Then, for the error $\tilde{e}(t)$ defined in (43), the following bound holds for all $m=1, \ldots, N$,

$$
\left\|\tilde{e}\left(t_{m}\right)\right\|_{L}^{2} \lesssim\left(E_{0}\right)^{2}+t_{m} \max _{1 \leq n \leq m}\left\{C_{n}^{\mathrm{T}}(u) \tau_{n}^{2(k+2)}+C_{n}^{\mathrm{S}}(u) h_{n}^{2 r+1}\right\}+C_{m}^{\prime}(u) h_{m}^{2(r+1)}+\left(E_{P, m}(u)\right)^{2},
$$

with $C_{n}^{\mathrm{T}}(u), C_{n}^{\mathrm{S}}(u)$ and $C_{m}^{\prime}(u)$ defined in Theorem 11 and $E_{0}:=\left\|P_{h}^{0} u_{0}-u_{0 h}\right\|_{L}$, while the cumulated projection error $E_{P, m}(u)$ is defined as

$$
\left(E_{P, m}(u)\right)^{2}:=\sum_{n=1}^{m}\left(E_{n}^{\mathrm{P}}(u)\right)^{2} \quad \text { with } E_{n}^{\mathrm{P}}(u) \text { defined in (59). }
$$

Moreover, assuming $\tau_{n} \lesssim \tau_{n-1}$ for all $n=2, \ldots, N$, the following bound holds:

$$
\|\tilde{e}\|_{L^{2}(I, L)}^{2} \lesssim\left(E_{0}\right)^{2}+T \max _{1 \leq n \leq N}\left\{C_{n}^{\mathrm{T}}(u) \tau_{n}^{2(k+2)}+C_{n}^{\mathrm{S}}(u) h_{n}^{2 r+1}\right\}+\left(E_{P, N}(u)\right)^{2} .
$$

Proof. Applying Lemma 8, we again infer that $\widetilde{B}_{h}^{n}\left(\tilde{e}_{\tau h}, v_{\tau h}\right)=-\widetilde{B}_{h}^{n}\left(\eta, v_{\tau h}\right)$, so that Lemma 16 leads to

$$
\widetilde{B}_{h}^{n}\left(\tilde{e}_{\tau h}, v_{\tau h}\right) \lesssim\left\{\left(E_{n}^{\mathrm{T}}(u)\right)^{2}+\left(E_{n}^{\mathrm{S}}(u)\right)^{2}\right\}^{1 / 2}\left\{Q_{n}\left(\left\|v_{\tau h}\right\|_{n}^{2}\right)\right\}^{1 / 2}+E_{n}^{\mathrm{P}}(u)\left\|\left[v_{\tau h}\right]_{n-1}\right\|_{L} .
$$

Since $\tilde{e}_{\tau h}\left(t_{n, \mu}\right) \in V_{h}^{n}$ for all right-sided Gauss-Radau integration points $t_{n, \mu} \in I_{n}$, the function $w=\tilde{e}_{\tau h}$ satisfies the asumption $w \in C^{0}(\bar{I}, L) \cap X_{\tau}^{k+1}(L) \cap \tilde{X}$ in Lemma 4 (stability property of $\widetilde{B}_{h}^{n}$ ) so that by setting $v_{\tau h}=I_{\tau}^{\mathrm{GR}} \tilde{e}_{\tau h} \in X_{\tau h}^{k}$, we infer the lower bound

$$
\widetilde{B}_{h}^{n}\left(\tilde{e}_{\tau h}, v_{\tau h}\right) \geq \frac{1}{2}\left\|\tilde{e}_{\tau h}\left(t_{n}\right)\right\|_{L}^{2}-\frac{1}{2}\left\|\tilde{e}_{\tau h}\left(t_{n-1}\right)\right\|_{L}^{2}+\frac{1}{2}\left\|\left[v_{\tau h}\right]_{n-1}\right\|_{L}^{2}+\alpha Q_{n}\left(\left\|\tilde{e}_{\tau h}\right\|_{n}^{2}\right) .
$$

The rest of the proof is similar to that of Theorem 11 and is skipped for brevity.

\subsubsection{Bound on the projection error}

Our goal is now to derive estimates on the cumulated projection error $E_{P, m}(u)$ for all $m=$ $1, \ldots, N$. Obviously, $E_{P, m}(u)=0$ if $V_{h}^{n} \subseteq V_{h}^{n-1}$ for all $n=1, \ldots, m$, i.e., if only mesh coarsenings occur from one time interval to the next one. To treat more general situations, we introduce some additional notation.

Let $n=1, \ldots, N$. We denote by $\mathcal{T}_{h}^{n \text {,coa }}$ the subset of the coarse mesh cells in $\mathcal{T}_{h}^{n}$ which are such that either they are in $\mathcal{T}_{h}^{n-1}$ or they can be decomposed by means of mesh cells from $\mathcal{T}_{h}^{n-1}$, and by $\mathcal{T}_{h}^{n \text {,ref }}$ the subset of the finer mesh cells in $\mathcal{T}_{h}^{n}$, i.e.,

$$
\mathcal{T}_{h}^{n, \text { coa }}:=\left\{K \in \mathcal{T}_{h}^{n} ; \exists \mathcal{T} \subset \mathcal{T}_{h}^{n-1}: \bar{K}=\bigcup_{T \in \mathcal{T}} \bar{T}\right\}, \quad \mathcal{T}_{h}^{n, \text { ref }}:=\mathcal{T}_{h}^{n} \backslash \mathcal{T}_{h}^{n, \text { coa }}
$$

We denote by $\Omega_{n}^{\text {ref }}$ the subset of the domain $\Omega$ where the mesh $\mathcal{T}_{h}^{n}$ is finer than $\mathcal{T}_{h}^{n-1}$, i.e., $\overline{\Omega_{n}^{\text {ref }}}:=\bigcup_{K \in \mathcal{T}_{h}^{n, \text { ref }}} \bar{K}$, and we set $h_{n}^{\text {ref }}:=\max _{K \in \mathcal{T}_{h}^{n, \text { ref }}} h_{K}$, while $\left|\Omega_{n}^{\text {ref }}\right|$ denotes the $d$-dimensional measure of $\Omega_{n}^{\text {ref }}$. 
We assume that the quasi-interpolation operator $\Pi_{h}^{n-1}$ has the property that

$$
\left.\left(v_{h}-\Pi_{h}^{n-1} v_{h}\right)\right|_{K}=0 \quad \forall v_{h} \in V_{h}^{n}, \forall K \in \mathcal{T}_{h}^{n, \text { coa }} .
$$

All the assumptions (56), (57) and (64) are satisfied if we choose for $\Pi_{h}^{n-1} v_{h}$ the standard Lagrange finite element interpolate of $v_{h} \in V_{h}^{n-1}+V_{h}^{n}$ defined on the mesh $\mathcal{T}_{h}^{n-1}$ in the case of conforming FEM or the $L^{2}$-orthogonal projection of $v_{h}$ onto the space $V_{h}^{n-1}$ in the case of DG methods where piecewise polynomial spaces are used. We first estimate the local projection error $E_{n}^{\mathrm{P}}(u)$ defined in (59).

Lemma 18 (Local projection error) The following bounds hold: In the case of DG spaces,

$$
E_{n}^{\mathrm{P}}(u) \lesssim\left|\Omega_{n}^{r e f}\right|^{1 / 2}\left(h_{n}^{r e f}\right)^{1 / 2}\left\{\left(h_{n}^{r e f}\right)^{r+1 / 2} C_{n}^{\mathrm{P}, \mathrm{S}}(u)\right\}
$$

with $C_{n}^{\mathrm{P}, \mathrm{S}}(u):=\left|u\left(t_{n-1}\right)\right|_{W^{r+1, \infty}\left(\Omega_{n}^{r e f}\right)}$, and in the case of conforming FEM, under assumption $(\boldsymbol{H})$,

$$
E_{n}^{\mathrm{P}}(u) \lesssim\left(h_{n}^{r e f}\right)^{1 / 2}\left\{\left(h_{n}\right)^{r+1 / 2} C_{n}^{\mathrm{P}, \mathrm{S}}(u)\right\}
$$

with $C_{n}^{\mathrm{P}, \mathrm{S}}(u):=\left|u\left(t_{n-1}\right)\right|_{H^{r+1}(\Omega)}$.

Proof. Owing to (64), we infer that $\left\|v_{h}-\Pi_{h}^{n-1} v_{h}\right\|_{L^{2}\left(\Omega \backslash \Omega_{n}^{\text {ref }}\right)}=0$ for all $v_{h} \in V_{h}^{n}$. Hence,

$$
E_{n}^{\mathrm{P}}(u) \leq\left\|u\left(t_{n-1}\right)-P_{h}^{n-1} u\left(t_{n-1}\right)\right\|_{L^{2}\left(\Omega_{n}^{\mathrm{ref}}\right)} .
$$

In the case of DG methods, we use the bound

$$
\left\|u\left(t_{n-1}\right)-P_{h}^{n-1} u\left(t_{n-1}\right)\right\|_{L^{2}\left(\Omega_{n}^{\mathrm{ref}}\right)} \leq\left|\Omega_{n}^{\mathrm{ref}}\right|^{1 / 2}\left\|u\left(t_{n-1}\right)-P_{h}^{n-1} u\left(t_{n-1}\right)\right\|_{L^{\infty}\left(\Omega_{n}^{\mathrm{ref}}\right)},
$$

and the local approximation properties of the $L^{2}$-orthogonal projection in $W^{r+1, \infty}\left(\Omega_{n}^{\text {ref }}\right)$ to infer (65). In the case of conforming FEM, we observe that

$$
\begin{aligned}
E_{n}^{\mathrm{P}}(u) & \leq\left(h_{n}^{\mathrm{ref}}\right)^{1 / 2}\left\|\mathfrak{h}^{-1 / 2}\left(u\left(t_{n-1}\right)-P_{h}^{n-1} u\left(t_{n-1}\right)\right)\right\|_{L^{2}\left(\Omega_{n}^{\text {ref }}\right)} \\
& \leq\left(h_{n}^{\text {ref }}\right)^{1 / 2}\left\|\mathfrak{h}^{-1 / 2}\left(u\left(t_{n-1}\right)-P_{h}^{n-1} u\left(t_{n-1}\right)\right)\right\|_{L^{2}(\Omega)},
\end{aligned}
$$

and proceed as in the proof of Lemma 1 to conclude.

Remark 19 (Comparison of (65) and (66)) In both estimates, the term between braces has the same order as the other terms stemming from space errors. Note, however, that for $D G$ methods, the constant $C_{n}^{\mathrm{P}, \mathrm{S}}(u)$ and the mesh size depend on $\Omega_{n}^{\text {ref }}$ only, while this dependency concerns the whole domain $\Omega$ for conforming FEM. Furthermore, the bound on the local projection error in the case of $D G$ spaces contains the additional factor $\left|\Omega_{n}^{\text {ref }}\right|^{1 / 2}$. In practical refinement regimes, a reasonable assumption is that

$$
\max _{n \in \mathcal{N}_{m}^{\text {ref }}}\left|\Omega_{n}^{\text {ref }}\right| \lesssim h_{n}^{\text {ref }}
$$


which is mostly due to the fact that singularities or critical parts of the exact solution are typically located in $(d-1)$-dimensional manifolds that are covered by a grid part with $d$ dimensional measure of order $h_{n}^{\text {ref }}$. In this situation, the bound on $E_{n}^{\mathrm{P}}(u)$ for $D G$ methods is improved by a factor $\left(h_{n}^{\text {ref }}\right)^{1 / 2}$ with respect to that for conforming FEM. The price to pay is a slightly more stringent regularity assumption on the exact solution. If assumption (67) cannot be exploited, both bounds (65) and (66) exhibit the same asymptotic behavior.

We now estimate the cumulated projection error. We define the index set $\mathcal{N}_{m}^{\text {ref }}$ of those $n \leq m$, where $V_{h}^{n}$ contains some refinement with respect to $V_{h}^{n-1}$, and the number $M_{m}$ of its elements, i.e.,

$$
\mathcal{N}_{m}^{\mathrm{ref}}:=\left\{n \in\{1, \ldots, m\} ; \quad V_{h}^{n} \nsubseteq V_{h}^{n-1}\right\}, \quad M_{m}:=\operatorname{card}\left(\mathcal{N}_{m}^{\mathrm{ref}}\right) .
$$

A straightforward consequence of Lemma 18 is the following result.

Corollary 20 (Cumulated projection error) The following bounds hold for all $m=1, \ldots, N$ : In the case of DG methods,

$$
\left|E_{P, m}(u)\right| \lesssim M_{m}^{1 / 2} \max _{n \in \mathcal{N}_{m}^{r e f}}\left\{\left|\Omega_{n}^{r e f}\right|^{1 / 2}\left(h_{n}^{r e f}\right)^{1 / 2}\left\{\left(h_{n}^{r e f}\right)^{r+1 / 2} C_{n}^{\mathrm{P}, \mathrm{S}}(u)\right\}\right\},
$$

and in the case of conforming FEM under assumption $(\boldsymbol{H})$,

$$
\left|E_{P, m}(u)\right| \lesssim M_{m}^{1 / 2} \max _{n \in \mathcal{N}_{m}^{r e f}}\left\{\left(h_{n}^{r e f}\right)^{1 / 2}\left\{\left(h_{n}\right)^{r+1 / 2} C_{n}^{\mathrm{P}, \mathrm{S}}(u)\right\}\right\} .
$$

Remark 21 (Interpretation of Corollary 20) Let $\tau_{a v, m}:=\frac{t_{m}}{m}$ denote the averaged time step in the interval $\left[0, t_{m}\right]$. Then, owing to the obvious estimate $M_{m} \leq m$, we infer in the case of DG methods that

$$
\left|E_{P, m}(u)\right| \lesssim t_{m}^{1 / 2}\left(\frac{\left(h_{(m)}^{r e f}\right)^{1+\alpha}}{\tau_{a v, m}}\right)^{1 / 2} \max _{n \in \mathcal{N}_{m}^{r e f}}\left\{\left(h_{n}^{r e f}\right)^{r+1 / 2} C_{n}^{\mathrm{P}, \mathrm{S}}(u)\right\},
$$

where $h_{(m)}^{\text {ref }}:=\max _{n \in \mathcal{N}_{m}^{\text {ref }}} h_{n}^{\text {ref }}$ and $\alpha=1$ if assumption (67) is valid, while $\alpha=0$ otherwise. This means that the projection error due to dynamic grids only causes a weakening of the overall order of accuracy if the averaged time step $\tau_{a v, m}$ is strongly less than $\left(h_{(m)}^{r e f}\right)^{1+\alpha}$. This is a mild restriction to practical regimes for controlling the ratio between mesh and time steps. In the case of conforming FEM, the bound (71) holds with $\alpha=0$ and $h_{n}$ in place of $h_{n}^{\text {ref }}$ in the term between braces.

\section{Estimates on error derivatives}

This section is devoted to estimating the time-derivative error in the $L$-norm and the error in the discrete graph norm. Throughout this section, we consider static meshes for simplicity, so that we drop the superscript $n$ on the mesh $\mathcal{T}_{h}$, the finite element space $V_{h}$, the discrete differential operator $A_{h}$, and the $L^{2}$-orthogonal projector $P_{h}$ onto $V_{h}$. In what follows, we assume that the exact solution $u$ has the regularity $u \in C^{1}(\bar{I}, W) \cap C^{2}(\bar{I}, L)$ (so that $f \in$ $\left.C^{1}(\bar{I}, L)\right)$. Moreover, we assume $k \geq 1$ as in Section 5 . 


\subsection{Time-derivative error estimates}

Our main idea is to derive a time-derivative error estimate by comparing the time-derivative of the exact solution with the post-processed time-derivative of the post-processed discrete solution. Since $\mathcal{L}_{\tau} u_{\tau h} \in X_{\tau}^{k+1}\left(V_{h}\right) \cap C^{0}\left(\bar{I}, V_{h}\right)$, the time-derivative of $\mathcal{L}_{\tau} u_{\tau h}$ is well-defined in the interior intervals $\left(t_{n-1}, t_{n}\right)$ and can be continuously extended from the left at all $t_{n}$ by setting

$$
\partial_{t} \mathcal{L}_{\tau} u_{\tau h}\left(t_{n}\right):=\lim _{t \uparrow t_{n}} \partial_{t} \mathcal{L}_{\tau} u_{\tau h}(t) \quad \forall n=1, \ldots, N .
$$

This defines the piecewise polynomial function $\partial_{t} \mathcal{L}_{\tau} u_{\tau h}$ over $I$, and at $t=0$, we define

$$
\partial_{t} \mathcal{L}_{\tau} u_{\tau h}(0):=P_{h} f(0)-A_{h} u_{\tau h}(0) .
$$

The function $\partial_{t} \mathcal{L}_{\tau} u_{\tau h}$ is now defined over $\bar{I}$, and $\partial_{t} \mathcal{L}_{\tau} u_{\tau h} \in X_{\tau h}^{k}$.

Lemma 22 The fully discrete solution $u_{\tau h}$ is such that

$$
\partial_{t} \mathcal{L}_{\tau} u_{\tau h}(t)+A_{h} u_{\tau h}(t)=P_{h} I_{\tau}^{\mathrm{GR}} f(t) \quad \forall t \in \bar{I},
$$

where the time-interpolate $I_{\tau}^{\mathrm{GR}} f$ of $f$ is defined as in (12).

Proof. Recalling (32), we obtain, for all $n=1, \ldots, N$ and all $v_{\tau h} \in \mathbb{P}_{k}\left(I_{n}, V_{h}\right)$,

$$
\int_{I_{n}}\left(\partial_{t} \mathcal{L}_{\tau} u_{\tau h}+A_{h} u_{\tau h}, v_{\tau h}\right)_{L} d t=Q_{n}\left(\left(f, v_{\tau h}\right)_{L}\right)=\int_{I_{n}}\left(I_{\tau}^{\mathrm{GR}} f, v_{\tau h}\right)_{L} d t=\int_{I_{n}}\left(P_{h} I_{\tau}^{\mathrm{GR}} f, v_{\tau h}\right)_{L} d t
$$

Hence, (74) holds in the interior of all time intervals. Moreover, (74) holds by definition at $t=0$, see $(73)$, and at all $t_{n}$ for all $n=1, \ldots, N$, owing to $(72)$ since both $A_{h} u_{\tau h}$ and $P_{h} I_{\tau}^{\mathrm{GR}} f$ are continuous from the left at $t_{n}$.

The error on the time-derivative can now be defined as

$$
\widehat{e}(t):=\partial_{t} u(t)-\mathcal{L}_{\tau} \partial_{t} \mathcal{L}_{\tau} u_{\tau h}(t) \quad \forall t \in \bar{I} .
$$

We observe that $\widehat{e}$ is continuous in time on $\bar{I}$.

Lemma 23 (Consistency) Assume $u \in C^{1}(\bar{I}, W) \cap C^{2}(\bar{I}, L)$. For all $n=1, \ldots, N$, the following identity holds:

$$
\widetilde{B}_{h}^{n}\left(\widehat{e}, v_{\tau h}\right)=Q_{n}\left(\left(\partial_{t} f-\partial_{t} L_{\tau}^{k+1} f, v_{\tau h}\right)_{L}\right) \quad \forall v_{\tau h} \in X_{\tau h}^{k},
$$

where $\left.L_{\tau}^{k+1} f\right|_{\bar{I}_{n}}$ is the Lagrange interpolate of $f$ in $\mathbb{P}_{k+1}\left(\bar{I}_{n}, L\right)$ based on the $(k+1)$ right-sided Gauss-Radau integration points on each $\bar{I}_{n}$ and the left endpoint $t_{n-1}$.

Proof. Since $\partial_{t}^{2} u(t)+A \partial_{t} u(t)=\partial_{t} f(t)$ for all $t \in I$, applying this equation at the $(k+1)$ right-sided Gauss-Radau integration points in $I_{n}$ and using the consistency $(20)$ of the discrete operator $A_{h}$, it is inferred that

$$
\widetilde{B}_{h}^{n}\left(\partial_{t} u, v_{\tau h}\right)=Q_{n}\left(\left(\partial_{t} f, v_{\tau h}\right)_{L}\right) .
$$


Moreover, applying the linear operator $\mathcal{L}_{\tau}$ to $(74)$ yields

$$
\mathcal{L}_{\tau} \partial_{t} \mathcal{L}_{\tau} u_{\tau h}(t)+A_{h} \mathcal{L}_{\tau} u_{\tau h}(t)=\mathcal{L}_{\tau} P_{h} I_{\tau}^{\mathrm{GR}} f(t) \quad \forall t \in \bar{I},
$$

where we have used that $\mathcal{L}_{\tau} A_{h} u_{\tau h}(t)=A_{h} \mathcal{L}_{\tau} u_{\tau h}(t)$. Taking the time-derivative, we infer that, for all $\mu=1, \ldots, k+1$ and all $n=1, \ldots, N$,

$$
\partial_{t} \mathcal{L}_{\tau} \partial_{t} \mathcal{L}_{\tau} u_{\tau h}\left(t_{n, \mu}\right)+\partial_{t} A_{h} \mathcal{L}_{\tau} u_{\tau h}\left(t_{n, \mu}\right)=\partial_{t} \mathcal{L}_{\tau} P_{h} I_{\tau}^{\mathrm{GR}} f\left(t_{n, \mu}\right),
$$

where we have taken the limit from the left at $t_{n, k+1}=t_{n}$. Recalling the definition (33) of the bilinear form $\widetilde{B}_{h}^{n}$, we obtain

$$
\begin{aligned}
\widetilde{B}_{h}^{n}\left(\mathcal{L}_{\tau} \partial_{t} \mathcal{L}_{\tau} u_{\tau h}, v_{\tau h}\right) & =Q_{n}\left(\left(\partial_{t} \mathcal{L}_{\tau} \partial_{t} \mathcal{L}_{\tau} u_{\tau h}+A_{h} \mathcal{L}_{\tau} \partial_{t} \mathcal{L}_{\tau} u_{\tau h}, v_{\tau h}\right)_{L}\right) \\
& =Q_{n}\left(\left(\partial_{t} \mathcal{L}_{\tau} \partial_{t} \mathcal{L}_{\tau} u_{\tau}+A_{h} \partial_{t} \mathcal{L}_{\tau} u_{\tau h}, v_{\tau h}\right)_{L}\right) \\
& =Q_{n}\left(\left(\partial_{t} \mathcal{L}_{\tau} \partial_{t} \mathcal{L}_{\tau} u_{\tau h}+\partial_{t} A_{h} \mathcal{L}_{\tau} u_{\tau h}, v_{\tau h}\right)_{L}\right) \\
& =Q_{n}\left(\left(\partial_{t} \mathcal{L}_{\tau} P_{h} I_{\tau}^{\mathrm{GR}} f, v_{\tau h}\right)_{L}\right)=Q_{n}\left(\left(\partial_{t} \mathcal{L}_{\tau} I_{\tau}^{\mathrm{GR}} f, v_{\tau h}\right)_{L}\right)
\end{aligned}
$$

where we have used (18) on the second line to drop the $\mathcal{L}_{\tau}$ operator after $A_{h}$, the fact that $A_{h}$ is time-independent on the third line, and dropped the projector $P_{h}$ on the last line since $P_{h}$ commutes with the operators $\mathcal{L}_{\tau}$ and $\partial_{t}$. To conclude, we observe that $L_{\tau}^{k+1} f=\mathcal{L}_{\tau} I_{\tau}^{\mathrm{GR}} f$ since the restrictions of both functions to $I_{n}$ are in $\mathbb{P}_{k+1}\left(I_{n}, L\right)$ and coincide at $(k+2)$ distinct points of $\bar{I}_{n}$.

Our analysis hinges on the following error decomposition:

$$
\widehat{e}(t)=\underbrace{\left(\partial_{t} u(t)-\mathcal{L}_{\tau} P_{h} \partial_{t} R_{\tau}^{k+1} u(t)\right)}_{=: \widehat{\eta}(t)}+\underbrace{\left(\mathcal{L}_{\tau} P_{h} \partial_{t} R_{\tau}^{k+1} u(t)-\mathcal{L}_{\tau} \partial_{t} \mathcal{L}_{\tau} u_{\tau h}(t)\right)}_{=: \widehat{e}_{\tau h}(t)} \quad \forall t \in \bar{I},
$$

where to define $\mathcal{L}_{\tau} P_{h} \partial_{t} R_{\tau}^{k+1} u(t)$ on the first time interval, we define $\partial_{t} R_{\tau}^{k+1} u(0):=\partial_{t} u(0)$. We observe that both $\widehat{\eta}$ and $\widehat{e}_{\tau h}$ are continuous functions in time on $\bar{I}$.

Lemma 24 (Boundedness) For all $n=1, \ldots, N$, the following bound holds:

$$
\left|\widetilde{B}_{h}^{n}\left(\widehat{\eta}, v_{\tau h}\right)\right| \lesssim\left\{\left(\widehat{E}_{n}^{\mathrm{T}}(u)\right)^{2}+\left(\widehat{E}_{n}^{\mathrm{S}}(u)\right)^{2}\right\}^{1 / 2}\left\{Q_{n}\left(\left\|v_{\tau h}\right\|^{2}\right)\right\}^{1 / 2}
$$

with the time and space errors respectively given by

$$
\begin{aligned}
& \left(\widehat{E}_{n}^{\mathrm{T}}(u)\right)^{2}:=Q_{n}\left(\left\|\partial_{t}\left(u-R_{\tau}^{k+1} u\right)\right\|_{V}^{2}+\left\|\partial_{t}^{2}\left(u-I_{\tau}^{k+2} u\right)\right\|_{L}^{2}\right), \\
& \left(\widehat{E}_{n}^{\mathrm{S}}(u)\right)^{2}:=Q_{n}\left(\left\|\partial_{t} R_{\tau}^{k+1} u-P_{h} \partial_{t} R_{\tau}^{k+1} u\right\|_{h, \frac{1}{2}}^{2}\right) .
\end{aligned}
$$

Proof. We decompose $\widetilde{B}_{h}^{n}\left(\widehat{\eta}, v_{\tau h}\right)$ as

$$
\begin{aligned}
\widetilde{B}_{h}^{n}\left(\widehat{\eta}, v_{\tau h}\right)= & Q_{n}\left(\left(\partial_{t}\left(\partial_{t} u-\mathcal{L}_{\tau} P_{h} \partial_{t} R_{\tau}^{k+1} u\right), v_{\tau h}\right)_{L}\right) \\
& +Q_{n}\left(\left(A_{h}\left(\partial_{t} u-\partial_{t} R_{\tau}^{k+1} u\right), v_{\tau h}\right)_{L}\right) \\
& +Q_{n}\left(\left(A_{h}\left(\partial_{t} R_{\tau}^{k+1} u-P_{h} \partial_{t} R_{\tau}^{k+1} u\right), v_{\tau h}\right)_{L}\right)=: T_{1}+T_{2}+T_{3},
\end{aligned}
$$


where we have used $Q_{n}\left(\left(A_{h} \mathcal{L}_{\tau} P_{h} \partial_{t} R_{\tau}^{k+1} u, v_{\tau h}\right)_{L}\right)=Q_{n}\left(\left(A_{h} P_{h} \partial_{t} R_{\tau}^{k+1} u, v_{\tau h}\right)_{L}\right)$ owing to (18). We first bound $T_{2}$ and $T_{3}$. Since $\left(\partial_{t} u-\partial_{t} R_{\tau}^{k+1} u\right)$ is in $W$, the consistency of the discrete operator $A_{h}$ together with the Cauchy-Schwarz inequality lead to

$$
\begin{aligned}
\left|T_{2}\right| & =\left|Q_{n}\left(\left(A\left(\partial_{t} u-\partial_{t} R_{\tau}^{k+1} u\right), v_{\tau h}\right)_{L}\right)\right| \\
& \lesssim\left\{Q_{n}\left(\left\|\partial_{t}\left(u-R_{\tau}^{k+1} u\right)\right\|_{V}^{2}\right)\right\}^{1 / 2}\left\{Q_{n}\left(\left\|v_{\tau h}\right\|_{L}^{2}\right)\right\}^{1 / 2} .
\end{aligned}
$$

For $T_{3}$, we use boundedness on orthogonal subscales (22) to infer that

$$
\left|T_{3}\right| \leq\left\{Q_{n}\left(\left\|\partial_{t} R_{\tau}^{k+1} u-P_{h} \partial_{t} R_{\tau}^{k+1} u\right\|_{h, \frac{1}{2}}^{2}\right)\right\}^{1 / 2}\left\{Q_{n}\left(\left\|v_{\tau h}\right\|^{2}\right)\right\}^{1 / 2} .
$$

Finally, concerning $T_{1}$, we observe that

$$
T_{1}=Q_{n}\left(\left(\partial_{t}^{2}\left(u-I_{\tau}^{k+2} u\right), v_{\tau h}\right)_{L}\right)+Q_{n}\left(\left(\partial_{t}\left\{\partial_{t} I_{\tau}^{k+2} u-\mathcal{L}_{\tau} P_{h} \partial_{t} R_{\tau}^{k+1} u\right\}, v_{\tau h}\right)_{L}\right)=: T_{1,1}+T_{1,2} .
$$

The term $T_{1,1}$ can be simply bounded as

$$
\left|T_{1,1}\right| \leq\left\{Q_{n}\left(\left\|\partial_{t}^{2}\left(u-I_{\tau}^{k+2} u\right)\right\|_{L}^{2}\right)\right\}^{1 / 2}\left\{Q_{n}\left(\left\|v_{\tau h}\right\|_{L}^{2}\right)\right\}^{1 / 2} .
$$

Turning to $T_{1,2}$, we can drop the projection $P_{h}$, and since both arguments of the $L^{2}$-inner product are in $\mathbb{P}_{k}\left(I_{n}, L\right)$, we infer that

$$
\begin{aligned}
T_{1,2} & =\int_{I_{n}}\left(\partial_{t}\left(\partial_{t} I_{\tau}^{k+2} u-\mathcal{L}_{\tau} \partial_{t} R_{\tau}^{k+1} u\right), v_{\tau h}\right)_{L} \\
& =-\int_{I_{n}}\left(\partial_{t} I_{\tau}^{k+2} u-\mathcal{L}_{\tau} \partial_{t} R_{\tau}^{k+1} u, \partial_{t} v_{\tau h}\right)_{L}+\left[\left(\partial_{t} I_{\tau}^{k+2} u-\mathcal{L}_{\tau} \partial_{t} R_{\tau}^{k+1} u, v_{\tau h}\right)_{L}\right]_{t_{n-1}^{+}}^{t_{n}}
\end{aligned}
$$

The first term on the right-hand side vanishes since the integrand is in $\mathbb{P}_{2 k}\left(I_{n}\right)$ and vanishes at all the right-sided Gauss-Radau integration points by construction. The contribution of the second term at $t_{n}$ vanishes for the same reason. Finally, the contribution of the second term at $t_{n-1}^{+}$also vanishes since

$$
\begin{aligned}
\partial_{t} I_{\tau}^{k+2} u\left(t_{n-1}^{+}\right)-\mathcal{L}_{\tau} \partial_{t} R_{\tau}^{k+1} u\left(t_{n-1}^{+}\right) & =\partial_{t} I_{\tau}^{k+2} u\left(t_{n-1}^{+}\right)-\partial_{t} R_{\tau}^{k+1} u\left(t_{n-1}\right) \\
& =\partial_{t} I_{\tau}^{k+2} u\left(t_{n-1}^{+}\right)-\partial_{t} I_{\tau}^{k+2} u\left(t_{n-1}\right)=0 .
\end{aligned}
$$

Collecting the above bounds yields the assertion.

Lemma 25 (Estimates on $\widehat{e}_{\tau h}$ ) For all $m=1, \ldots, N$, the following bound holds:

$$
\left\|\widehat{e}_{\tau h}\left(t_{m}\right)\right\|_{L}^{2}+\sum_{n=1}^{m} Q_{n}\left(\left\|\widehat{e}_{\tau h}\right\|^{2}\right) \lesssim\left(\widehat{E}_{0}\right)^{2}+\sum_{n=1}^{m}\left\{\left(\widehat{E}_{n}^{\mathrm{T}}(u)\right)^{2}+\left(\widehat{E}_{n}^{\mathrm{S}}(u)\right)^{2}+\left(\widehat{E}_{n}^{\mathrm{R}}(f)\right)^{2}\right\},
$$

with right-hand side error $\left(\widehat{E}_{n}^{\mathrm{R}}(f)\right)^{2}:=Q_{n}\left(\left\|\partial_{t} f-\partial_{t} L_{\tau}^{k+1} f\right\|_{L}^{2}\right)$ and initial error $\left(\widehat{E}_{0}\right)^{2}:=$ $\left.\| P_{h} A u_{0}-A_{h} u_{0 h}\right) \|_{L}^{2}$. Moreover, assuming $\tau_{n} \lesssim \tau_{n-1}$ and $\tau_{1} \lesssim 1$, the following bound holds:

$$
\left\|\widehat{e}_{\tau h}\right\|_{L^{2}(I, L)}^{2} \lesssim\left(\widehat{E}_{0}\right)^{2}+\sum_{n=1}^{N}\left\{\left(\widehat{E}_{n}^{\mathrm{T}}(u)\right)^{2}+\left(\widehat{E}_{n}^{\mathrm{S}}(u)\right)^{2}+\left(\widehat{E}_{n}^{\mathrm{R}}(f)\right)^{2}\right\} .
$$


Proof. Owing to Lemma 23 (consistency) and the error decomposition (77), we infer that

$$
\widetilde{B}_{h}^{n}\left(\widehat{e}_{\tau h}, v_{\tau h}\right)=-\widetilde{B}_{h}^{n}\left(\widehat{\eta}, v_{\tau h}\right)+Q_{n}\left(\left(\partial_{t} f-\partial_{t} L_{\tau}^{k+1} f, v_{\tau h}\right)_{L}\right) .
$$

The first term on the right-hand side is bounded using Lemma 24 and the second one using the Cauchy-Schwarz inequality, yielding

$$
\left.\widetilde{B}_{h}^{n}\left(\widehat{e}_{\tau h}, v_{\tau h}\right) \lesssim\left\{\left(\widehat{E}_{n}^{\mathrm{T}}(u)\right)^{2}+\left(\widehat{E}_{n}^{\mathrm{S}}(u)\right)^{2}+\widehat{E}_{n}^{\mathrm{R}}(f)\right)^{2}\right\}^{1 / 2}\left\{Q_{n}\left(\left\|v_{\tau h}\right\|^{2}\right)\right\}^{1 / 2}
$$

Setting $v_{\tau h}=I_{\tau}^{\mathrm{GR}} \widehat{e}_{\tau h}$ and using the stability property of $\widetilde{B}_{h}^{n}$ stated in Lemma 4 for the time-continuous function $\widehat{e}_{\tau h}$ together with a Young inequality, we infer, as in the proof of Lemma 10, that

$$
\left.\frac{1}{2}\left\|\widehat{e}_{\tau h}\left(t_{n}\right)\right\|_{L}^{2}-\frac{1}{2}\left\|\widehat{e}_{\tau h}\left(t_{n-1}\right)\right\|_{L}^{2}+Q_{n}\left(\left\|\widehat{e}_{\tau h}\right\|^{2}\right) \lesssim\left(\widehat{E}_{n}^{\mathrm{T}}(u)\right)^{2}+\left(\widehat{E}_{n}^{\mathrm{S}}(u)\right)^{2}+\widehat{E}_{n}^{\mathrm{R}}(f)\right)^{2} .
$$

Moreover, the initial discrete error is $\widehat{e}_{\tau h}(0)=P_{h} \partial_{t} u(0)-\partial_{t} \mathcal{L}_{\tau} u_{\tau h}(0)=-P_{h} A u_{0}+A_{h} u_{0 h}$. We conclude as in the proof of Lemma 10.

Theorem 26 (Time-derivative error estimate) Let $u$ be the exact solution and let $u_{\tau h}$ be the fully discrete solution. Assume $k \geq 1$. Assume $(\boldsymbol{H})$ in the case of conforming FEM. Then, for the error $\widehat{e}(t)$ defined in (75), the following bound holds for all $m=1, \ldots, N$,

$$
\left\|\widehat{e}\left(t_{m}\right)\right\|_{L}^{2} \lesssim\left(\widehat{E}_{0}\right)^{2}+t_{m} \max _{1 \leq n \leq m}\left\{\widehat{C}_{n}^{\mathrm{T}}(u, f) \tau_{n}^{2(k+1)}+C_{n}^{\mathrm{S}}(u) h^{2 r+1}\right\}+\widehat{C}_{m}^{\prime}(u) h^{2(r+1)},
$$

with $\widehat{C}_{n}^{\mathrm{T}}(u, f):=C_{n}^{\mathrm{T}}(u)+|f|_{C^{k+2}\left(\bar{I}_{n}, L\right)}^{2}, C_{n}^{\mathrm{T}}(u)$ and $C_{n}^{\mathrm{S}}(u)$ defined in Theorem 11, and $\widehat{C}_{m}^{\prime}(u)=$ $\left|\partial_{t} u\left(t_{m}\right)\right|_{H^{r+1}(\Omega)}^{2}$. Moreover, assuming $\tau_{n} \lesssim \tau_{n-1}$ for all $n=2, \ldots, N$ and $\tau_{1} \lesssim 1$, the following bound holds:

$$
\|\widehat{e}\|_{L^{2}(I, L)}^{2} \lesssim\left(\widehat{E}_{0}\right)^{2}+T \max _{1 \leq n \leq N}\left\{\widehat{C}_{n}^{\mathrm{T}}(u, f) \tau_{n}^{2(k+1)}+C_{n}^{\mathrm{S}}(u) h^{2 r+1}\right\} .
$$

Proof. We infer that, for all $n=1, \ldots, N$,

$$
\begin{aligned}
\left|\widehat{E}_{n}^{\mathrm{T}}(u)\right|^{2} & \lesssim \tau_{n}\left(\tau_{n}^{2(k+1)}|u|_{C^{k+3}\left(\bar{I}_{n}, L\right)}^{2}+\tau_{n}^{2(k+1)}|u|_{C^{k+2}\left(\bar{I}_{n}, V\right)}^{2}\right), \\
\left|\widehat{E}_{n}^{\mathrm{S}}(u)\right|^{2} & \lesssim \tau_{n} h^{2 r+1}\|u\|_{C^{1}\left(\bar{I}_{n}, H^{r+1}(\Omega)\right)}^{2}, \\
\left|\widehat{E}_{n}^{\mathrm{R}}(f)\right|^{2} & \lesssim \tau_{n} \tau_{n}^{2(k+1)}|f|_{C^{k+2}\left(\bar{I}_{n}, L\right)}^{2} .
\end{aligned}
$$

using (38) with $B=L$ and (42) with $B=V$ for the time error, the approximation property (29) of $P_{h}$ combined with the stability of $R_{\tau}^{k+1}$ from Corollary 7 with $B=H^{r+1}(\Omega)$ for the space error, and the approximation properties of the Lagrange interpolate $L_{\tau}^{k+1}$ for the right-hand side error. Moreover, recalling the error decomposition (77), we observe that, for all $m=1, \ldots, N$,

$$
\left\|\widehat{\eta}\left(t_{m}\right)\right\|_{L}=\left\|\partial_{t} u\left(t_{m}\right)-P_{h} \partial_{t} u\left(t_{m}\right)\right\|_{L} \lesssim h^{r+1}\left|\partial_{t} u\right|_{H^{r+1}(\Omega)},
$$


since $\vartheta_{m}\left(t_{m}\right)=1$ and $\partial_{t} R_{\tau}^{k+1} u\left(t_{m}\right)=\partial_{t} u\left(t_{m}\right)$. In addition, using the definition of $\mathcal{L}_{\tau}$ and the triangle inequality, we infer that

$$
\|\widehat{\eta}\|_{L^{2}\left(I_{n}, L\right)}^{2} \leq 2\left\|\partial_{t} u-P_{h} \partial_{t} R_{\tau}^{k+1} u\right\|_{L^{2}\left(I_{n}, L\right)}^{2}+2\left\|P_{h}\left[\partial_{t} R_{\tau}^{k+1} u\right]_{n-1} \vartheta_{n}\right\|_{L^{2}\left(I_{n}, L\right)}^{2}=: T_{1}+T_{2}
$$

Using again the triangle inequality, the fact that $P_{h}$ is a projection, and (42) with $B=L$ leads to

$$
\begin{aligned}
\left|T_{1}\right| & \lesssim\left\|\partial_{t} u-P_{h} \partial_{t} u\right\|_{L^{2}\left(I_{n}, L\right)}^{2}+\left\|\partial_{t} u-\partial_{t} R_{\tau}^{k+1} u\right\|_{L^{2}\left(I_{n}, L\right)}^{2} \\
& \lesssim \tau_{n}\left(h^{2(r+1)}|u|_{C^{1}\left(\bar{I}_{n}, H^{r+1}(\Omega)\right)}^{2}+\tau_{n}^{2(k+1)}|u|_{C^{k+2}\left(\bar{I}_{n}, L\right)}^{2}\right),
\end{aligned}
$$

while using the regularity of $u$, the fact that $\partial_{t} R_{\tau}^{k+1} u\left(t_{n-1}\right)=\partial_{t} u\left(t_{n-1}\right)$, and (42) with $B=L$ leads to

$$
\begin{aligned}
\left|T_{2}\right| & \lesssim \tau_{n}\left\|\left[\partial_{t} R_{\tau}^{k+1} u\right]_{n-1}\right\|_{L}^{2}=\tau_{n}\left\|\partial_{t} R_{\tau}^{k+1} u\left(t_{n-1}^{+}\right)-\partial_{t} u\left(t_{n-1}\right)\right\|_{L}^{2} \\
& \leq \tau_{n}\left\|\partial_{t} u-\partial_{t} R_{\tau}^{k+1} u\right\|_{C^{0}\left(\bar{I}_{n}, L\right)}^{2} \lesssim \tau_{n} \tau_{n}^{2(k+1)}|u|_{C^{k+2}\left(\bar{I}_{n}, L\right)}^{2} .
\end{aligned}
$$

We conclude using Lemma 25 and the triangle inequality, as well as $h \lesssim 1$ and $\|\cdot\|_{L} \lesssim\|\cdot\|_{V}$ for $(84)$.

Remark 27 (Initial error) The initial error $\widehat{E}_{0}$ vanishes when the discrete initial condition is chosen as the solution of the steady transport problem $A_{h} u_{0 h}=P_{h} A u_{0}$. Otherwise, this error is typically of order $h^{r}$ if $u_{0}$ is smooth enough.

\subsection{Discrete graph norm error estimates}

Lemma 28 (Graph norm estimate on $\tilde{e}_{\tau h}$ ) For all $m=1, \ldots, N$, the following bound holds:

$$
\sum_{n=1}^{m} Q_{n}\left(\left\|\tilde{e}_{\tau h}\right\|_{\sharp}^{2}\right) \lesssim\left(\widehat{E}_{0}\right)^{2}+\sum_{n=1}^{m}\left\{\left(E_{n}^{\mathrm{T}}(u)\right)^{2}+\left(E_{n}^{\mathrm{S}}(u)\right)^{2}+\left(\widehat{E}_{n}^{\mathrm{T}}(u)\right)^{2}+\left(\widehat{E}_{n}^{\mathrm{S}}(u)\right)^{2}+\left(\widehat{E}_{n}^{\mathrm{R}}(f)\right)^{2}\right\} .
$$

Proof. Owing to the discrete inf-sup condition satisfied by $A_{h}$, see (24), we know that, for all $n=1, \ldots, N$ and all $\mu=1, \ldots, k+1$, there is $w_{n, \mu} \in V_{h}$ such that

$$
\left\|\tilde{e}_{\tau h}\left(t_{n, \mu}\right)\right\|_{\sharp}^{2} \lesssim\left(A_{h} \tilde{e}_{\tau h}\left(t_{n, \mu}\right), w_{n, \mu}\right)_{L} \quad \text { and } \quad\left\|w_{n, \mu}\right\|_{\sharp} \leq\left\|\tilde{e}_{\tau h}\left(t_{n, \mu}\right)\right\|_{\sharp} .
$$

Let $w_{\tau h}$ be the function in $X_{\tau h}^{k}$ uniquely defined by $w_{\tau h}\left(t_{n, \mu}\right):=w_{n, \mu}$, for all $n=1, \ldots, N$ and all $\mu=1, \ldots, k+1$. Then, we obtain

$$
Q_{n}\left(\left\|\tilde{e}_{\tau h}\right\|_{\sharp}^{2}\right) \lesssim Q_{n}\left(\left(A_{h} \tilde{e}_{\tau h}, w_{\tau h}\right)_{L}\right) \quad \text { and } \quad Q_{n}\left(\left\|w_{\tau h}\right\|_{\sharp}^{2}\right) \leq Q_{n}\left(\left\|\tilde{e}_{\tau h}\right\|_{\sharp}^{2}\right) .
$$

Using the definition of $\widetilde{B}_{h}^{n}$ and consistency (Lemma 8), we obtain

$$
\begin{aligned}
Q_{n}\left(\left(A_{h} \tilde{e}_{\tau h}, w_{\tau h}\right)_{L}\right) & =\widetilde{B}_{h}^{n}\left(\tilde{e}_{\tau h}, w_{\tau h}\right)-Q_{n}\left(\left(\partial_{t} \tilde{e}_{\tau h}, w_{\tau h}\right)_{L}\right) \\
& =-\widetilde{B}_{h}^{n}\left(\eta, w_{\tau h}\right)-Q_{n}\left(\left(\partial_{t} \tilde{e}_{\tau h}, w_{\tau h}\right)_{L}\right) \\
& =-\widetilde{B}_{h}^{n}\left(\eta, w_{\tau h}\right)-Q_{n}\left(\left(\widehat{e}_{\tau h}, w_{\tau h}\right)_{L}\right),
\end{aligned}
$$


since it is readily deduced from (45) and (77) that $\widehat{e}_{\tau h}\left(t_{n, \mu}\right)=\mathcal{L}_{\tau} \partial_{t} \tilde{e}_{\tau h}\left(t_{n, \mu}\right)=\partial_{t} \tilde{e}_{\tau h}\left(t_{n, \mu}\right)$ for all $n=1, \ldots, N$ and all $\mu=1, \ldots, k+1$. The first term on the right-hand side is bounded using Lemma 9 and the second term using the Cauchy-Schwarz inequality, yielding

$$
Q_{n}\left(\left\|\tilde{e}_{\tau h}\right\|_{\sharp}^{2}\right) \lesssim\left\{\left(E_{n}^{\mathrm{T}}(u)\right)^{2}+\left(E_{n}^{\mathrm{S}}(u)\right)^{2}+Q_{n}\left(\left\|\widehat{e}_{\tau h}\right\|_{L}^{2}\right)\right\}^{1 / 2}\left\{Q_{n}\left(\left\|w_{\tau h}\right\|^{2}\right)\right\}^{1 / 2} .
$$

Observing that $Q_{n}\left(\left\|w_{\tau h}\right\|^{2}\right) \leq Q_{n}\left(\left\|w_{\tau h}\right\|_{\sharp}^{2}\right) \leq Q_{n}\left(\left\|\tilde{e}_{\tau h}\right\|_{\sharp}^{2}\right)$, we obtain by Young's inequality

$$
Q_{n}\left(\left\|\tilde{e}_{\tau h}\right\|_{\sharp}^{2}\right) \lesssim\left(E_{n}^{\mathrm{T}}(u)\right)^{2}+\left(E_{n}^{\mathrm{S}}(u)\right)^{2}+Q_{n}\left(\left\|\widehat{e}_{\tau h}\right\|_{L}^{2}\right) .
$$

We conclude summing from $n=1$ to $m$, and using Lemma 25 to bound $Q_{n}\left(\left\|\widehat{e}_{\tau h}\right\|_{L}^{2}\right)$.

Theorem 29 (Graph norm error estimate) Let $u$ be the exact solution and let $u_{\tau h}$ be the fully discrete solution. Assume $k \geq 1$ and $\tau_{n} \lesssim 1$ for all $n=1, \ldots, N$. Assume $(\boldsymbol{H})$ in the case of conforming FEM. Then, for the error $\tilde{e}(t)$ defined in (43) and all $m=1, \ldots, N$, the following bound holds:

$$
\sum_{n=1}^{m} Q_{n}\left(\|\tilde{e}\|_{\sharp}^{2}\right) \lesssim\left(\widehat{E}_{0}\right)^{2}+t_{m} \max _{1 \leq n \leq m}\left\{\widetilde{C}_{n}^{\mathrm{T}}(u, f) \tau_{n}^{2(k+1)}+C_{n}^{\mathrm{S}}(u) h^{2 r+1}\right\},
$$

where $\widetilde{C}_{n}^{\mathrm{T}}(u, f):=|u|_{C^{k+3}\left(\bar{I}_{n}, L\right)}^{2}+|u|_{C^{k+2}\left(\bar{I}_{n}, W\right)}^{2}+|f|_{C^{k+2}\left(\bar{I}_{n}, L\right)}^{2}$ and $C_{n}^{\mathrm{S}}(u)$ defined in Theorem 11.

Proof. Recalling the error decomposition (45) and using the triangle inequality, we need to bound $\sum_{n=1}^{m} Q_{n}\left(\left\|\tilde{e}_{\tau h}\right\|_{\sharp}^{2}\right)$ and $\sum_{n=1}^{m} Q_{n}\left(\|\eta\|_{\sharp}^{2}\right)$. For the first term, we use Lemma 28 together with the bounds on the various errors derived in the proofs of Theorems 11 and 26 to infer that $\sum_{n=1}^{m} Q_{n}\left(\left\|\tilde{e}_{\tau h}\right\|_{\sharp}^{2}\right)$ is bounded by the right-hand side of (86) (observe in particular for the time error that $\|\cdot\|_{V} \lesssim\|\cdot\|_{W}$ so that $\left.\widehat{C}_{n}^{\mathrm{T}}(u, f) \lesssim \widetilde{C}_{n}^{\mathrm{T}}(u, f)\right)$. Concerning the second term, we use the triangle inequality, Lemma 6 (with $B=W$ ), the fact that $\|w\|_{\sharp} \lesssim\|w\|_{W}$ for all $w \in W$, the approximation property of $P_{h}$ in the $\|\cdot\|_{\sharp}$-norm, and the stability of $R_{\tau}^{k+1}$ (with $B=H^{r+1}(\Omega)$ ) to infer that

$$
\begin{aligned}
Q_{n}\left(\|\eta\|_{\sharp}^{2}\right) & \leq 2 Q_{n}\left(\left\|u-R_{\tau}^{k+1} u\right\|_{\sharp}^{2}\right)+2 Q_{n}\left(\left\|R_{\tau}^{k+1} u-P_{h} R_{\tau}^{k+1} u\right\|_{\sharp}^{2}\right) \\
& \lesssim \tau_{n}^{2(k+2)}|u|_{C^{k+2}\left(\bar{I}_{n}, W\right)}^{2(2 r+1}+h^{2 r+1}\|u\|_{C^{1}\left(\bar{I}_{n}, H^{r+1}(\Omega)\right)}^{2} .
\end{aligned}
$$

Summing over $n$ and recalling that $\tau_{n} \lesssim 1$ yields the assertion.

\section{Appendix}

Proof of Lemma 6. Let $\hat{I}:=[-1,1]$ denote the reference time interval and $T_{n}: \hat{I} \rightarrow \bar{I}_{n}$ the affine reference mapping with $t=T_{n}(\hat{t}):=\left(t_{n-1}+t_{n}\right) / 2+\frac{\tau_{n}}{2} \hat{t}$. We assign to $u \in C^{k+2}\left(\bar{I}_{n}, B\right)$ a reference function $\hat{u} \in C^{k+2}(\hat{I}, B)$ defined by $\hat{u}(\hat{t}):=\left.u\right|_{I_{n}}\left(T_{n}(\hat{t})\right)$ for all $\hat{t} \in \hat{I}$, where we take the right-sided limit $u\left(t_{n-1}^{+}\right)$for $\hat{t}=-1$. Then, for $m=1, \ldots, k+2$, we infer that

$$
\partial_{\hat{t}}^{m} \hat{u}(\hat{t})=\partial_{t}^{m} u\left(T_{n}(\hat{t})\right)\left(\frac{\tau_{n}}{2}\right)^{m} \quad \forall \hat{t} \in \hat{I},
$$


where for boundary points $\hat{t}= \pm 1$ the one-sided derivatives of $u$ are taken that come from interval $I_{n}$. The idea of the proof is to construct an interpolation operator $\hat{R}_{\tau}^{k+1}: C^{1}(\hat{I}, B) \rightarrow$ $\mathbb{P}_{k+1}(\hat{I}, B)$, which is related to $R_{\tau}^{k+1}$ by

$$
\hat{R}_{\tau}^{k+1} \hat{u}(\hat{t})=R_{\tau}^{k+1} u(t) \quad \forall \hat{t} \in \hat{I}, \quad t=T_{n}(\hat{t}),
$$

and satisfies the properties

$$
\begin{aligned}
\hat{R}_{\tau}^{k+1} \hat{p} & =\hat{p} & & \forall \hat{p} \in \mathbb{P}_{k+1}(\hat{I}, B) . \\
\left\|\hat{R}_{\tau}^{k+1} \hat{w}\right\|_{C^{0}(\hat{I}, B)} & \leq M\|\hat{w}\|_{C^{1}(\hat{I}, B)} & & \forall \hat{w} \in C^{1}(\hat{I}, B) .
\end{aligned}
$$

Let $\hat{p} \in \mathbb{P}_{k+1}(\hat{I}, B)$ be the Taylor polynomial $\hat{p}(\hat{t}):=\sum_{j=0}^{k+1} \frac{1}{j !} \partial_{\hat{t}}^{j} \hat{u}(0) \hat{t}^{j}$. Since $\partial_{\hat{t}} \hat{p}$ is the Taylor polynomial for $\partial_{\hat{t}} \hat{u}$ of order $k$, we infer that

$$
\|\hat{u}-\hat{p}\|_{C^{1}(\hat{I}, B)} \leq \frac{1}{(k+1) !}|\hat{u}|_{C^{k+2}(\hat{I}, B)}=\frac{\tau_{n}^{k+2}}{2^{k+2}(k+1) !}|u|_{C^{k+2}\left(\bar{I}_{n}, B\right)}
$$

Then, using (88) and (89) leads to

$$
\begin{aligned}
\left\|u-R_{\tau}^{k+1} u\right\|_{C^{0}\left(\bar{I}_{n}, B\right)} & =\left\|\hat{u}-\hat{R}_{\tau}^{k+1} \hat{u}\right\|_{C^{0}(\hat{I}, B)} \leq\|\hat{u}-\hat{p}\|_{C^{0}(\hat{I}, B)}+\left\|\hat{R}_{\tau}^{k+1}(\hat{p}-\hat{u})\right\|_{C^{0}(\hat{I}, B)} \\
& \leq(1+M)\|\hat{u}-\hat{p}\|_{C^{1}(\hat{I}, B)} \lesssim \tau_{n}^{k+2}|u|_{C^{k+2}\left(\bar{I}_{n}, B\right)},
\end{aligned}
$$

yielding (41), while the stability of $R_{\tau}^{k+1}$ results from (89) and the use of the reference mapping. It remains to construct the operator $\hat{R}_{\tau}^{k+1}$ and to verify (87), (88), and (89). Let $\hat{\varphi}_{j} \in \mathbb{P}_{k+2}(\hat{I}), j=0, \ldots, k+2$, denote the basis functions of the Lagrange/Hermite interpolation with respect to the nodal points $-1=\hat{s}_{0}<\cdots<\hat{s}_{k}=1$ such that the interpolate $\hat{I}_{\tau}^{k+2} \hat{u} \in \mathbb{P}_{k+2}(\hat{I}, B)$ verifying $\hat{I}_{\tau}^{k+2} \hat{u}\left(\hat{s}_{j}\right)=\hat{u}\left(\hat{s}_{j}\right), j=0, \ldots, k$, and $\partial_{\hat{t}}\left(\hat{I}_{\tau}^{k+2} \hat{u}\right)( \pm 1)=\partial_{\hat{t}} \hat{u}( \pm 1)$ has the representation

$$
\hat{I}_{\tau}^{k+2} \hat{u}=\sum_{j=0}^{k} \hat{u}\left(\hat{s}_{j}\right) \hat{\varphi}_{j}+\partial_{\hat{t}} \hat{u}(-1) \hat{\varphi}_{k+1}+\partial_{\hat{t}} \hat{u}(1) \hat{\varphi}_{k+2} .
$$

This representation implies the stability estimate

$$
\left\|\hat{I}_{\mathcal{\tau}}^{k+2} \hat{u}\right\|_{C^{1}(\hat{I}, B)} \leq M_{1}\|\hat{u}\|_{C^{1}(\hat{I}, B)} \quad \text { with } \quad M_{1}:=\sum_{j=0}^{k+2}\left\|\hat{\varphi}_{j}\right\|_{C^{1}(\hat{I}, \mathbb{R})} .
$$

Moreover, $\hat{I}_{\tau}^{k+2} \hat{u}(\hat{t})=I_{\tau}^{k+2} u\left(T_{n}(\hat{t})\right)$ for all $\hat{t} \in \hat{I}$. Now, let $\hat{\phi}_{j} \in \mathbb{P}_{k}(\hat{I}), j=1, \ldots, k+1$, denote the basis functions of the Lagrange interpolation with respect to the right-sided Gauss-Radau integration points $\hat{t}_{\mu} \in \hat{I}, \mu=1, \ldots, k+1$, satisfying the property $\hat{\phi}_{j}\left(\hat{t}_{\mu}\right)=\delta_{j, \mu}$ for all $j, \mu \in\{1, \ldots, k+1\}$ where $\delta_{j, \mu}$ denotes Kronecker symbol. Using these basis functions, we define functions $\hat{\psi}_{j} \in \mathbb{P}_{k+1}(\hat{I}), j=0, \ldots, k+1$, by means of

$$
\hat{\psi}_{0}(\hat{t}):=1, \quad \hat{\psi}_{j}(\hat{t}):=\int_{-1}^{\hat{t}} \hat{\phi}_{j}(s) d s \quad \forall j=1, \ldots, k+1 .
$$


These functions satisfy the properties $\partial_{\hat{t}} \hat{\psi}_{j}\left(\hat{t}_{\mu}\right)=\delta_{j, \mu}$ for all $j=0, \ldots, k+1$ and $\mu=1, \ldots, k+1$ as well as $\hat{\psi}_{j}(-1)=\delta_{j, 0}$ for all $j=0, \ldots, k+1$. Therefore, the functions $\hat{\psi}_{j}$ form a basis of $\mathbb{P}_{k+1}(\hat{I})$ and the interpolate

$$
\hat{R}_{\tau}^{k+1} \hat{u}:=\hat{I}_{\tau}^{k+2} \hat{u}(-1) \hat{\psi}_{0}+\sum_{j=1}^{k+1} \partial_{\hat{t}} \hat{I}_{\tau}^{k+2} \hat{u}\left(\hat{t}_{j}\right) \hat{\psi}_{j}
$$

satisfies the conditions

$$
\hat{R}_{\mathcal{\tau}}^{k+1} \hat{u}(-1)=\hat{I}_{\tau}^{k+2} \hat{u}(-1), \quad \partial_{\hat{t}} \hat{R}_{\tau}^{k+1} \hat{u}\left(\hat{t}_{\mu}\right)=\partial_{\hat{t}} \hat{I}_{\tau}^{k+2} \hat{u}\left(\hat{t}_{\mu}\right) \quad \forall \mu=1, \ldots, k+1 .
$$

This shows that (87) holds since the polynomial $R_{\tau}^{k+1} u\left(T_{n}(\hat{t})\right)$ is also in $\mathbb{P}_{k+1}(\hat{I}, B)$ and satisfies the above conditions owing to (39)-(40). Applying the definition (91) to an arbitrary $\hat{w} \in C^{1}(\hat{I}, B)$, we obtain by means of $(90)$ the stability estimate

$$
\left\|\hat{R}_{\tau}^{k+1} \hat{w}\right\|_{C^{0}(\hat{I}, B)} \leq\left\|\hat{I}_{\tau}^{k+2} \hat{w}\right\|_{C^{1}(\hat{I}, B)} \sum_{j=0}^{k+1}\left\|\hat{\psi}_{j}\right\|_{C^{0}(\hat{I}, \mathbb{R})} \leq M\|\hat{w}\|_{C^{1}(\hat{I}, B)},
$$

where $M=M_{1} \sum_{j=0}^{k+1}\left\|\hat{\psi}_{j}\right\|_{C^{0}(\hat{I}, \mathbb{R})}$ which proves (89). Finally, to prove (88), we observe that an arbitrary polynomial $\hat{p} \in \mathbb{P}_{k+1}(\hat{I}, B)$ has a basis representation of the form

$$
\hat{p}(\hat{t})=\sum_{j=0}^{k+1} \hat{p}_{j} \hat{\psi}_{j}(\hat{t}) \quad \forall \hat{t} \in \hat{I}, \quad \hat{p}_{j} \in B .
$$

From the properties of the basis functions $\hat{\psi}_{j}$, we obtain $\hat{p}(-1)=\hat{p}_{0}$ and $\partial_{\hat{t}} \hat{p}\left(\hat{t}_{j}\right)=\hat{p}_{j}$ for all $j=1, \ldots, k+1$. Since $\hat{I}_{\tau}^{k+2}$ leaves $\mathbb{P}_{k+1}(\hat{I}, B)$ invariant, we infer that $\hat{p}=\hat{p}(-1) \hat{\psi}_{0}+$ $\sum_{j=1}^{k+1} \partial_{\hat{t}} \hat{p}\left(\hat{t}_{j}\right) \hat{\psi}_{j}=\hat{R}_{\tau}^{k+1} \hat{p}$.

\section{References}

[1] E. Bänsch A. Brenner and M. Bause. A priori error analysis for finite element approximations of the stokes problem on dynamic meshes. IMA J. Numer. Anal., 34(1):123-146, 2014.

[2] N. Ahmed, G. Matthies, L. Tobiska, and H. Xie. Discontinuous Galerkin time stepping with local projection stabilization for transient convection-diffusion-reaction problems. Comput. Methods Appl. Mech. Engrg., 200(21-22):1747-1756, 2011.

[3] R. E. Bank and H. Yserentant. On the $H^{1}$-stability of the $L_{2}$-projection onto finite element spaces. Numer. Math., 126:361-381, 2014.

[4] M. Braack, E. Burman, V. John, and G. Lube. Stabilized finite element methods for the generalized Oseen problem. Comput. Methods Appl. Mech. Engrg., 196(4-6):853-866, 2007.

[5] F. Brezzi, L. D. Marini, and E. Süli. Discontinuous Galerkin methods for first-order hyperbolic problems. Math. Models Methods Appl. Sci., 14(12):1893-1903, 2004.

[6] E. Burman. A unified analysis for conforming and nonconforming stabilized finite element methods using interior penalty. SIAM J. Numer. Anal., 43(5):2012-2033 (electronic), 2005.

[7] E. Burman and A. Ern. A continuous finite element method with face penalty to approximate Friedrichs' systems. M2AN Math. Model. Numer. Anal., 41(1):55-76, 2007. 
[8] E. Burman, A. Ern, and M.A. Fernández. Explicit Runge-Kutta schemes and finite elements with symmetric stabilization for first-order linear PDE systems. SIAM J. Numer. Anal., 48(6):2019-2042, 2010.

[9] E. Burman and P. Hansbo. Edge stabilization for Galerkin approximations of convection-diffusionreaction problems. Comput. Methods Appl. Mech. Engrg., 193:1437-1453, 2004.

[10] J. Česenek and M. Feistauer. Theory of the space-time discontinuous Galerkin method for nonstationary parabolic problems with nonlinear convection and diffusion. SIAM J. Numer. Anal., 50(3):1181-1206, 2012.

[11] B. Cockburn, S. Hou, and C.-W. Shu. The Runge-Kutta local projection discontinuous Galerkin finite element method for conservation laws. IV. The multidimensional case. Math. Comp., 54(190):545-581, 1990.

[12] B. Cockburn, S. Lin, and C.-W. Shu. TVB Runge-Kutta local projection discontinuous Galerkin finite element method for conservation laws. III. One-dimensional systems. J. Comput. Phys., 84(1):90-113, 1989.

[13] B. Cockburn and C.-W. Shu. TVB Runge-Kutta local projection discontinuous Galerkin finite element method for conservation laws. II. General framework. Math. Comp., 52(186):411-435, 1989.

[14] R. Codina. Stabilization of incompressibility and convection through orthogonal sub-scales in finite element methods. Comput. Methods Appl. Mech. Engrg., 190(13-14):1579-1599, 2000.

[15] R. Codina. Stabilized finite element approximation of transient incompressible flows using orthogonal subscales. Comput. Methods Appl. Mech. Engrg., 191(39-40):4295-4321, 2002.

[16] D. A. Di Pietro and A. Ern. Mathematical Aspects of Discontinuous Galerkin Methods, volume 69 of Mathématiques \& Applications. Springer, Berlin Heidelberg, 2012.

[17] K. Eriksson and C. Johnson. Adaptive finite element methods for parabolic problems. II. Optimal error estimates in $L_{\infty} L_{2}$ and $L_{\infty} L_{\infty}$. SIAM J. Numer. Anal., 32(3):706-740, 1995.

[18] A. Ern and J.-L. Guermond. Theory and practice of finite elements, volume 159 of Applied Mathematical Sciences. Springer-Verlag, New York, 2004.

[19] A. Ern and J.-L. Guermond. Discontinuous Galerkin methods for Friedrichs' systems. I. General theory. SIAM J. Numer. Anal., 44(2):753-778, 2006.

[20] M. Feistauer, V. Kučera, K. Najzar, and J. Prokopová. Analysis of space-time discontinuous Galerkin method for nonlinear convection-diffusion problems. Numer. Math., 117(2):251-288, 2011.

[21] K. O. Friedrichs. Symmetric positive linear differential equations. Comm. Pure and Appl. Math., 11:333$418,1958$.

[22] J.-L. Guermond. Stabilization of Galerkin approximations of transport equations by subgrid modeling. M2AN Math. Model. Numer. Anal., 33(6):1293-1316, 1999.

[23] J.-L. Guermond. Subgrid stabilization of Galerkin approximations of linear monotone operators. IMA J. Numer. Anal., 21:165-197, 2001.

[24] M. Hochbruck and T. Pažur. Implicit Runge-Kutta methods and discontinuous Galerkin discretizations for linear Maxwell's equations. Technical report, Karlsruhe Institute of Technology, 2013.

[25] S. Hussain, F. Schieweck, and S. Turek. Higher order Galerkin time discretizations and fast multigrid solvers for the heat equation. J. Numer. Math., 19(1):41-61, 2011.

[26] S. Hussain, F. Schieweck, and S. Turek. Higher order Galerkin time discretization for nonstationary incompressible flow. Technical report, Fakultät für Mathematik, TU Dortmund, January 2012. Ergebnisberichte des Instituts für Angewandte Mathematik, Nummer 444.

[27] S. Hussain, F. Schieweck, and S. Turek. A note on accurate and efficient higher order Galerkin time stepping schemes for the nonstationary Stokes equations. The Open Numerical Methods Journal, 4:35-45, 2012.

[28] J. Jaffré, C. Johnson, and A. Szepessy. Convergence of the discontinuous Galerkin finite element method for hyperbolic conservation laws. Math. Models Methods Appl. Sci., 5(3):367-386, 1995. 
[29] C. Johnson and J. Pitkäranta. An analysis of the discontinuous Galerkin method for a scalar hyperbolic equation. Math. Comp., 46(173):1-26, 1986.

[30] P. Lesaint and P.-A. Raviart. On a finite element method for solving the neutron transport equation. In Mathematical Aspects of Finite Elements in Partial Differential Equations, pages 89-123. Publication No. 33. Math. Res. Center, Univ. of Wisconsin-Madison, Academic Press, New York, 1974.

[31] D. Leykekhman and B. Vexler. Optimal a priori error estimates of parabolic optimal control problems with pointwise control. SIAM J. Numer. Anal., 51(5):2797-2821, 2013.

[32] G. Matthies and F. Schieweck. Higher order variational time discretizations for nonlinear systems of ordinary differential equations. Preprint 23/2011, Otto-von-Guericke Universität Magdeburg, Fakultät für Mathematik, 2011. http://www-ian.math.uni-magdeburg.de/home/schieweck.

[33] T. Richter, A. Springer, and B. Vexler. Efficient numerical realization of discontinuous Galerkin methods for temporal discretization of parabolic problems. Numer. Math., 124(1):151-182, 2013.

[34] H.-G. Roos, M. Stynes, and L. Tobiska. Robust numerical methods for singularly perturbed differential equations, volume 24 of Springer Series in Computational Mathematics. Springer-Verlag, Berlin, second edition, 2008. Convection-diffusion-reaction and flow problems.

[35] D. Schötzau and C. Schwab. An $h p$ a priori error analysis of the DG time-stepping method for initial value problems. Calcolo, 37(4):207-232, 2000.

[36] A. Springer and B. Vexler. Third order convergent time discretization for parabolic optimal control problems with control constraints. Comput. Optim. Appl., 57(1):205-240, 2014.

[37] V. Thomée. Galerkin finite element methods for parabolic problems, volume 25 of Springer Series in Computational Mathematics. Springer-Verlag, Berlin, second edition, 2006.

[38] J. J. W. van der Vegt and H. van der Ven. Space-time discontinuous Galerkin finite element method with dynamic grid motion for inviscid compressible flows. I. General formulation. J. Comput. Phys., 182(2):546-585, 2002.

[39] T. Werder, K. Gerdes, D. Schötzau, and C. Schwab. hp-discontinuous Galerkin time stepping for parabolic problems. Comput. Methods Appl. Mech. Engrg., 190(49-50):6685-6708, 2001.

[40] D. Yang. Improved error estimation of dynamic finite element methods for second-order parabolic equations. J. Comput. Appl. Math., 126(1-2):319-338, 2000.

[41] K. Yosida. Functional Analysis. Classics in Mathematics. Springer-Verlag, Berlin, Germany, 1995. Reprint of the sixth (1980) edition.

[42] Q. Zhang and C.-W. Shu. Error estimates to smooth solutions of Runge-Kutta discontinuous Galerkin methods for scalar conservation laws. SIAM J. Numer. Anal., 42(2):641-666 (electronic), 2004.

[43] Q. Zhang and C.-W. Shu. Stability analysis and a priori error estimates of the third order explicit RungeKutta discontinuous Galerkin method for scalar conservation laws. SIAM J. Numer. Anal., 48(3):1038$1063,2010$. 